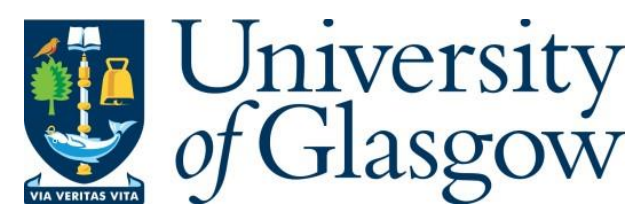

Li, X. (2017) Partial transformation groupoids attached to graphs and semigroups. International Mathematics Research Notices, 2017(17), pp. 5233-5259.

There may be differences between this version and the published version. You are advised to consult the publisher's version if you wish to cite from it.

http://eprints.gla.ac.uk/210210/

Deposited on: 25 February 2020

Enlighten - Research publications by members of the University of Glasgow http://eprints.gla.ac.uk 


\title{
PARTIAL TRANSFORMATION GROUPOIDS ATTACHED TO GRAPHS AND SEMIGROUPS
}

\begin{abstract}
XIN LI
Abstract. We introduce the notion of continuous orbit equivalence for partial dynamical systems, and give an equivalent characterization in terms of Cartan-isomorphisms for partial $\mathrm{C}^{*}$ crossed products. Both graph $\mathrm{C}^{*}$-algebras and semigroup $\mathrm{C}^{*}$-algebras can be described as $\mathrm{C}^{*}$ algebras attached to partial dynamical systems. As applications, for graphs, we generalize and explain a result of Matsumoto and Matui relating orbit equivalence and Cartan-isomorphism, and for semigroups, we strengthen several structural results for semigroup $\mathrm{C}^{*}$-algebras concerning amenability, nuclearity as well as simplicity of boundary quotients. We also discuss pure infiniteness for partial transformation groupoids arising from graphs and semigroups.
\end{abstract}

\section{INTRODUCTION}

Recently, in the setting of ordinary topological dynamical systems, the notion of continuous orbit equivalence was introduced, and a $\mathrm{C}^{*}$-algebraic characterization was given involving Cartanisomorphisms (see [21]). We present a generalization to partial dynamical systems. This step is important as many $\mathrm{C}^{*}$-algebras appear naturally as crossed products attached to partial dynamical systems, while the setting of ordinary dynamical systems is more restricted. Our main motivation stems from graph $\mathrm{C}^{*}$-algebras and semigroup $\mathrm{C}^{*}$-algebras, both of which can be described in a very natural way as $\mathrm{C}^{*}$-algebras of partial dynamical systems. This description turns out to be very helpful for the study of structural properties of these $\mathrm{C}^{*}$-algebras.

In the case of graphs, we obtain a very easy explanation why all graph $\mathrm{C}^{*}$-algebras are nuclear (Remark 3.6). This goes back to the observation that non-abelian free semigroups embed into amenable groups. Furthermore, we generalize and explain results in $[24,25]$ about orbit equivalence and Cartan isomorphism from shifts of finite type to general graphs (Theorem 3.9). We note that a generalization of the results in [25] has been established independently in [3].

For semigroup $\mathrm{C}^{*}$-algebras and their boundary quotients, we are able to generalize several structural results from [19]. The key idea is that using partial transformation groupoids, we obtain structural results for our $\mathrm{C}^{*}$-algebras without assuming independence or the Toeplitz condition, which were crucial in our previous approach (see $[18,19])$. We obtain general characterizations for nuclearity of semigroup $\mathrm{C}^{*}$-algebras (Theorem 3.15) and simplicity of boundary quotients (Theorem 3.22).

2010 Mathematics Subject Classification. Primary 46L05; Secondary 37B05, 37A20.

Research supported by EPSRC grant EP/M009718/1. 
Furthermore, we study which partial transformation groupoids of graphs and semigroups are purely infinite, in the sense of [27]. In the case of graphs, we are able to prove that the partial system of a graph is residually topologically free and purely infinite if and only if the corresponding graph $\mathrm{C}^{*}$-algebra is purely infinite (Corollary 4.4). For semigroups, we show that the partial transformation groupoid corresponding to the boundary quotient is purely infinite if and only if the semigroup is not left reversible (Theorem 4.5). We are also able to identify a class of integral domains whose $a x+b$-semigroups have purely infinite partial transformation groupoids (Theorem 4.6). These results strengthen and explain previous results in [19, 20]. Finally, we close with a remark (Proposition 4.7) on almost finite groupoids in the sense of [26, $\S 6]$.

\section{Partial actions, transformation groupoids, $\mathrm{C}^{*}$-algebras and Cartan SUBALGEBRAS}

In the following, groups are discrete and countable, and topological spaces are locally compact, Hausdorff and second countable.

Definition 2.1. Let $G$ be a group with identity e, and let $X$ be a topological space. A partial action $\alpha$ of $G$ on $X$ consists of

- a collection $\left\{U_{g}\right\}_{g \in G}$ of open subsets $U_{g} \subseteq X$,

- a collection $\left\{\alpha_{g}\right\}_{g \in G}$ of homeomorphisms $\alpha_{g}: U_{g^{-1}} \rightarrow U_{g}, x \mapsto g . x$ such that $-U_{e}=X, \alpha_{e}=\mathrm{id}_{X}$

- for all $g_{1}, g_{2} \in G$, we have $g_{2} \cdot\left(U_{\left(g_{1} g_{2}\right)^{-1}} \cap U_{g_{2}^{-1}}\right)=U_{g_{2}} \cap U_{g_{1}^{-1}}$, and $\left(g_{1} g_{2}\right) \cdot x=g_{1} \cdot\left(g_{2} \cdot x\right)$ for all $x \in U_{\left(g_{1} g_{2}\right)^{-1}} \cap U_{g_{2}^{-1}}$.

We call such a triple $(X, G, \alpha)$ a partial system, and denote it by $\alpha: G \curvearrowright X$ or simply $G \curvearrowright X$.

Let $\alpha: G \curvearrowright X$ be a partial system. The dual action $\alpha^{*}$ of $\alpha$ is the partial action (in the sense of [28]) of $G$ on $C_{0}(X)$ given by $\alpha_{g}^{*}: C_{0}\left(U_{g^{-1}}\right) \rightarrow C_{0}\left(U_{g}\right), f \mapsto f \circ g^{-1}$.

The transformation groupoid attached to the partial system $\alpha: G \curvearrowright X$ is given by

$$
G_{\alpha} \ltimes X:=\left\{(g, x) \in G \times X: g \in G, x \in U_{g^{-1}}\right\},
$$

with source map $s(g, x)=x$, range map $r(g, x)=g \cdot x$, composition $\left(g_{1}, g_{2} . x\right)\left(g_{2}, x\right)=\left(g_{1} g_{2}, x\right)$ and inverse $(g, x)^{-1}=\left(g^{-1}, g \cdot x\right)$. We equip $G_{\alpha} \ltimes X$ with the subspace topology from $G \times X$. Usually, we write $G \ltimes X$ for $G_{\alpha} \ltimes X$ if the action $\alpha$ is understood. The unit space of $G \ltimes X$ coincides with $X$. Since $G$ is discrete, $G \ltimes X$ is an étale groupoid. Actually, if we set $G_{x}:=$ $\left\{g \in G: x \in U_{g^{-1}}\right\}$ and $G^{x}:=\left\{g \in G: x \in U_{g}\right\}$ for $x \in X$, then we have canonical identifications $s^{-1}(x) \cong G_{x},(g, x) \mapsto g$ and $r^{-1}(x) \cong G^{x},\left(g, g^{-1} \cdot x\right) \mapsto g$.

Let us now recall the construction (from [28]) of the reduced crossed product $C_{0}(X) \rtimes_{\alpha^{*}, r} G$ attached to our partial system $\alpha: G \curvearrowright X$. As with groupoids, we omit $\alpha^{*}$ in our notation for the crossed product. First of all, $C_{0}(X) \rtimes^{\ell^{1}} G:=\left\{\sum_{g} f_{g} \delta_{g} \in \ell^{1}\left(G, C_{0}(X)\right): f_{g} \in C_{0}\left(U_{g}\right)\right\}$ becomes a *algebra under component-wise addition, multiplication given by $\left(\sum_{g} f_{g} \delta_{g}\right) \cdot\left(\sum_{h} \tilde{f}_{h} \delta_{h}\right):=$ 
$\sum_{g, h} \alpha_{g}^{*}\left(\alpha_{g^{-1}}^{*}\left(f_{g}\right) \tilde{f}_{h}\right) \delta_{g h}$ and involution $\left(\sum_{g} f_{g} \delta_{g}\right)^{*}:=\sum_{g} \alpha_{g}^{*}\left(f_{g^{-1}}^{*}\right) \delta_{g}$, for $f_{g} \in C_{0}\left(U_{g}\right)$ and $\tilde{f}_{h} \in$ $C_{0}\left(U_{h}\right)$.

As in [28], we construct a representation of $C_{0}(X) \rtimes^{\ell^{1}} G$. Viewing $X$ as a discrete set, we define $\ell^{2} X$ and the representation $M: C_{0}(X) \rightarrow \mathcal{L}\left(\ell^{2} X\right), f \mapsto M(f)$, where $M(f)$ is the multiplication operator $M(f)(\xi):=f \cdot \xi$ for $\xi \in \ell^{2} X$. M is obviously a faithful representation of $C_{0}(X)$. Every $g \in G$ leads to a twist of $M$, namely $M_{g}: C_{0}(X) \rightarrow \mathcal{L}\left(\ell^{2} X\right)$ given by $M_{g}(f) \xi:=\left.\left(\left.f\right|_{U_{g}} \circ g\right) \cdot \xi\right|_{U_{g-1}}$. Here we view $\left.f\right|_{U_{g}} \circ g$ as an element in $C_{b}\left(U_{g^{-1}}\right)$, and $C_{b}\left(U_{g^{-1}}\right)$ acts on $\ell^{2} U_{g^{-1}}$ just by multiplication operators. Given $\xi \in \ell^{2} X$, we set $\left.\xi\right|_{U_{g^{-1}}}(x):=\xi(x)$ if $x \in U_{g^{-1}}$ and $\left.\xi\right|_{U_{g^{-1}}}(x):=0$ if $x \notin U_{g^{-1}}$. In other words, $\left.\xi\right|_{U_{g^{-1}}}$ is the component of $\xi$ in $\ell^{2} U_{g^{-1}}$ with respect to the decomposition $\ell^{2} X=\ell^{2} U_{g^{-1}} \oplus \ell^{2} U_{g^{-1}}^{c}$. So we have $M_{g}(f) \xi(x)=f(g . x) \xi(x)$ if $x \in U_{g^{-1}}$ and $M_{g}(f) \xi(x)=0$ if $x \notin U_{g^{-1}}$.

Consider now the Hilbert space $H:=\ell^{2}\left(G, \ell^{2} X\right) \cong \ell^{2} G \otimes \ell^{2} X$, and define the representation $\mu: C_{0}(X) \rightarrow \mathcal{L}(H)$ given by $\mu(f)\left(\delta_{g} \otimes \xi\right):=\delta_{g} \otimes M_{g}(f) \xi$. For $g \in G$, let $E_{g}$ be the orthogonal projection onto $\overline{\mu\left(C_{0}\left(U_{g^{-1}}\right)\right) H}$. Moreover, let $\lambda$ denote the left regular representation of $G$ on $\ell^{2} G$, and set $V_{g}:=\left(\lambda_{g} \otimes I\right) \cdot E_{g}$. Here $I$ is the identity operator on $H$.

We can now define the representation $\mu \times \lambda: C_{0}(X) \rtimes^{\ell^{1}} G \rightarrow \mathcal{L}(H), \sum_{g} f_{g} \delta_{g} \mapsto \sum_{g} \mu\left(f_{g}\right) V_{g}$. Following the original definition in [28], we set $C_{0}(X) \rtimes_{r} G:=\overline{C_{0}(X) \rtimes^{\ell^{1}} G} \|^{\|\cdot\|_{\mu \times \lambda}}$.

The following result follows from [1, Theorem 3.3], but we give a direct and short proof.

Proposition 2.2. The canonical homomorphism

$$
C_{c}(G \ltimes X) \rightarrow C_{0}(X) \rtimes^{\ell^{1}} G, \theta \mapsto \sum_{g} \theta\left(g, g^{-1} \cdot \sqcup\right) \delta_{g},
$$

where $\theta\left(g, g^{-1} . \sqcup\right)$ is the function $U_{g^{-1}} \rightarrow \mathbb{C}, x \mapsto \theta\left(g, g^{-1} \cdot x\right)$, extends to an isomorphism $C_{r}^{*}(G \ltimes$ $X) \stackrel{\cong}{\longrightarrow} C_{0}(X) \rtimes_{r} G$.

Proof. As above, let $\mu \times \lambda$ be the representation $C_{0}(X) \rtimes^{\ell^{1}} G \rightarrow \mathcal{L}(H)$ which we used to define $C_{0}(X) \rtimes_{r} G$. Our first observation is

$$
\overline{\operatorname{Im}(\mu \times \lambda)(H)}=\bigoplus_{h \in G} \delta_{h} \otimes \ell^{2} U_{h^{-1}}
$$

To see this, observe that for all $g \in G, \operatorname{Im}\left(E_{g}\right) \subseteq \bigoplus_{h} \delta_{h} \otimes \ell^{2}\left(U_{h^{-1}} \cap U_{(g h)^{-1}}\right)$. This holds since for $x \notin h^{-1}$. $\left(U_{h} \cap U_{g^{-1}}\right)=U_{(g h)^{-1}} \cap U_{h^{-1}},\left.f\right|_{U_{h}}(h . x)=0$ for $f \in C_{0}\left(U_{g^{-1}}\right)$. Therefore, $\pi\left(C_{0}\left(U_{g^{-1}}\right)\right)\left(\delta_{h} \otimes \ell^{2} X\right) \subseteq \delta_{h} \otimes \ell^{2}\left(U_{h^{-1}} \cap U_{(g h)^{-1}}\right)$. Hence $\operatorname{Im}\left(E_{g}\right) \subseteq \bigoplus_{h} \delta_{h} \otimes \ell^{2}\left(U_{h^{-1}} \cap U_{(g h)^{-1}}\right)$, and thus, $\operatorname{Im}\left(V_{g}\right) \subseteq \bigoplus_{h} \delta_{g h} \otimes \ell^{2}\left(U_{h^{-1}} \cap U_{(g h)^{-1}}\right) \subseteq \bigoplus_{h} \delta_{h} \otimes \ell^{2} U_{h^{-1}}$. This shows " $\subseteq$ " in (2). For "卫", note that for $f \in C_{0}(X),(\mu \times \lambda)\left(f \delta_{e}\right)=\mu(f) E_{e}$, and for $\xi \in \ell^{2} U_{h^{-1}}, \mu(f) E_{e}\left(\delta_{h} \otimes \xi\right)=$ $\delta_{h} \otimes\left(\left.f\right|_{U_{h}} \circ h\right) \xi$. So $(\mu \times \lambda)\left(f \delta_{e}\right)(H)$ contains $\delta_{h} \otimes f \cdot \xi$ for all $f \in C_{0}\left(U_{h^{-1}}\right)$ and $\xi \in \ell^{2} U_{h^{-1}}$, hence also $\delta_{h} \otimes \ell^{2} U_{h^{-1}}$. This proves " $\supseteq$ ".

For $x \in X$, let $G_{x}=\left\{g \in G: x \in U_{g^{-1}}\right\}$ as before. Our second observation is that for every $x \in X$, the subspace $H_{x}:=\ell^{2} G_{x} \otimes \delta_{x}$ is $(\mu \times \lambda)$-invariant. It is clear that $\mu(f)$ leaves $H_{x}$ invariant for all $f \in C_{0}(X)$. For $g, h \in G, E_{g}\left(\delta_{h} \otimes \delta_{x}\right)=\delta_{h} \otimes \delta_{x}$ if $x \in U_{h^{-1}} \cap U_{(g h)^{-1}}$, and if that is the case, then $V_{g}\left(\delta_{h} \otimes \delta_{x}\right)=\delta_{g h} \otimes \delta_{x} \in H_{x}$. 
Therefore, $H=\left(\bigoplus_{x \in X} H_{x}\right) \oplus(\mu \times \lambda)\left(C_{0}(X) \rtimes^{\ell^{1}} G\right)(H)^{\perp}$ is a decomposition of $H$ into $\mu \times \lambda$ invariant subspaces. For $x \in X$, set $\rho_{x}:=\left.(\mu \times \lambda)\right|_{H_{x}}$. Then $C_{0}(X) \rtimes_{r} G=\overline{C_{0}(X) \rtimes^{\ell^{1}} G} \|^{\|\cdot\|_{\oplus_{x} \rho_{x}}}$. Moreover, we have for $x \in U_{h^{-1}}$,

$$
\begin{aligned}
\rho_{x}\left(\sum_{g} f_{g} \delta_{g}\right)\left(\delta_{h} \otimes \delta_{x}\right) & =\sum_{g} \mu\left(f_{g}\right) V_{g}\left(\delta_{h} \otimes \delta_{x}\right)=\sum_{g: x \in U_{(g h)^{-1}}} \mu\left(f_{g}\right)\left(\delta_{g h} \otimes \delta_{x}\right) \\
& =\sum_{g: x \in U_{(g h)^{-1}}} \delta_{g h} \otimes f_{g}(g h . x) \delta_{x}=\sum_{k \in G_{x}} \delta_{k} \otimes f_{k h^{-1}}(k . x) \delta_{x} .
\end{aligned}
$$

Let us now compare this construction with the construction of the reduced groupoid $\mathrm{C}^{*}$-algebra of $G \ltimes X$. Obviously, (1) is an embedding of $C_{c}(G \ltimes X)$ as a subalgebra which is $\|\cdot\|_{\ell^{1}}$-dense in $C_{0}(X) \rtimes^{\ell^{1}} G$. Therefore, $C_{0}(X) \rtimes_{r} G={\overline{C_{c}(G \ltimes X)}}^{\|\cdot\|_{\oplus_{x} \rho_{x}}}$.

Now, to construct the reduced groupoid $\mathrm{C}^{*}$-algebra $C_{r}^{*}(G \ltimes X)$, we follow [35, § 2.3.4] and construct for every $x \in X$ the representation $\pi_{x}: C_{c}(G \ltimes X) \rightarrow \mathcal{L}\left(\ell^{2}\left(s^{-1}(x)\right)\right)$ by setting $\pi_{x}(\theta)(\xi)(\zeta):=\sum_{\eta \in s^{-1}(x)} \theta\left(\zeta \eta^{-1}\right) \xi(\eta)$. In our case, using $s^{-1}(x)=G_{x} \times\{x\}$, we obtain for $\xi=\delta_{h} \otimes \delta_{x}$ with $h \in G_{x}: \pi_{x}(\theta)\left(\delta_{h} \otimes \delta_{x}\right)(k, x)=\theta\left((k, x)(h, x)^{-1}\right)=\theta\left(k h^{-1}, h . x\right)$. Thus,

$$
\pi_{x}(\theta)\left(\delta_{h} \otimes \delta_{x}\right)(k, x)=\sum_{k \in G_{x}} \theta\left(k h^{-1}, h . x\right) \delta_{k} \otimes \delta_{x} .
$$

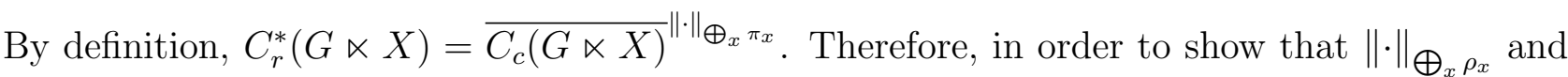
$\|\cdot\|_{\oplus_{x} \pi_{x}}$ coincide on $C_{c}(G \ltimes X)$, it suffices to show that for every $x \in X, \pi_{x}$ and the restriction of $\rho_{x}$ to $C_{c}(G \ltimes X)$ are unitarily equivalent. Given $x \in X$, using $s^{-1}(x)=G_{x} \times\{x\}$, we obtain the canonical unitary $\ell^{2}\left(s^{-1}(x)\right) \cong H_{x}=\ell^{2}\left(G_{x}\right) \otimes \delta_{x}$, so that we may think of both $\rho_{x}$ and $\pi_{x}$ as representations on $\ell^{2}\left(G_{x}\right) \otimes \delta_{x}$. We then have for $x \in X, \theta \in C_{c}(G \ltimes X)$ and $h \in G_{x}$ :

$$
\rho_{x}(\theta)\left(\delta_{h} \otimes \delta_{x}\right) \stackrel{(1)}{=} \rho_{x}\left(\sum_{g} \theta\left(g, g^{-1} . \sqcup\right) \delta_{g}\right)\left(\delta_{h} \otimes \delta_{x}\right) \stackrel{(3)}{=} \sum_{k \in G_{x}} \delta_{k} \otimes \theta\left(k h^{-1}, h . x\right) \delta_{x} \stackrel{(4)}{=} \pi_{x}(\theta)\left(\delta_{h} \otimes \delta_{x}\right) .
$$

This yields the canonical identification $C_{0}(X) \rtimes_{r} G \cong C_{r}^{*}(G \ltimes X)$, as desired.

Following [14], we define topological freeness as follows:

Definition 2.3. A partial system $G \curvearrowright X$ is called topologically free if for every $e \neq g \in G$, $\left\{x \in U_{g^{-1}}: g . x \neq x\right\}$ is dense in $U_{g^{-1}}$.

Lemma 2.4. A partial system $G \curvearrowright X$ is topologically free if and only if $\left\{x \in X: g . x \neq x\right.$ for all $\left.e \neq g \in G_{x}\right\}$ is dense in $X$.

Proof. The direction " $\Leftarrow$ " is simple: If $\left\{x \in X: g . x \neq x\right.$ for all $\left.e \neq g \in G_{x}\right\}$ is dense in $X$, then in particular, for every fixed $e \neq g \in G$, the set $\left\{x \in X: g . x \neq x\right.$ if $\left.g \in G_{x}\right\}$ is dense in $X$. Hence $\left\{x \in U_{g^{-1}}: g . x \neq x\right\}=\left\{x \in X: g . x \neq x\right.$ if $\left.g \in G_{x}\right\} \cap U_{g^{-1}}$ is dense in $U_{g^{-1}}$.

For " $\Rightarrow$ ", note that because of topological freeness, we know that for every $e \neq g \in G$, the open set $\left\{x \in U_{g^{-1}}: g . x \neq x\right\} \cup{\overline{U_{g^{-1}}}}^{c}$ is dense in $X$. Therefore,

$$
\left\{x \in X: g . x \neq x \text { for all } e \neq g \in G_{x}\right\}=\bigcap_{e \neq g \in G}\left\{x \in X: g . x \neq x \text { if } g \in G_{x}\right\}
$$


must be dense in $X$ since it contains $\bigcap_{e \neq g \in G}\left(\left\{x \in U_{g^{-1}}: g \cdot x \neq x\right\} \cup{\overline{U_{g^{-1}}}}^{c}\right)$ which is dense in $X$ by the Baire category theorem.

Corollary 2.5. A partial system $G \curvearrowright X$ is topologically free if and only if the transformation groupoid $G \ltimes X$ is topologically principal.

Recall (see [34]) that a topological groupoid $\mathcal{G}$ is called topologically principal if the set of points in $\mathcal{G}^{(0)}$ with trivial isotropy is dense in $\mathcal{G}^{(0)}$. Here, $x \in \mathcal{G}^{(0)}$ is said to have trivial isotropy if for all $\gamma \in \mathcal{G}, s(\gamma)=t(\gamma)=x$ already implies $\gamma=x$.

Proof. In the case of the transformation groupoid $\mathcal{G}=G \ltimes X, x \in X$ has trivial isotropy if whenever $g \in G$ satisfies $g \in G_{x}$ and $g . x=x$, then we must have $g=e$. Hence the set of points with trivial isotropy is nothing else but $\left\{x \in X: g . x \neq x\right.$ for all $\left.e \neq g \in G_{x}\right\}$. With this observation, our corollary follows immediately from Lemma 2.4.

Let us now introduce the notion of continuous orbit equivalence for partial systems, generalizing [21, Definition 2.5].

Definition 2.6. Partial systems $G \curvearrowright X$ and $H \curvearrowright Y$ are called continuously orbit equivalent if there exists a homeomorphism $\varphi: X \stackrel{\cong}{\longrightarrow} Y$ and continuous maps $a: \bigcup_{g \in G}\{g\} \times U_{g^{-1}} \rightarrow H$, $b: \bigcup_{h \in H}\{h\} \times V_{h^{-1}} \rightarrow G$ (where $V_{h^{-1}}$ is the domain of the partial homeomorphism attached to $h \in H$ ) such that

$$
\begin{aligned}
\varphi(g . x) & =a(g, x) \cdot \varphi(x), \\
\varphi^{-1}(h . y) & =b(h, y) \cdot \varphi^{-1}(y) .
\end{aligned}
$$

Implicitly, we require here that $a(g, x) \in H_{\varphi(x)}$ and $b(h, y) \in G_{\varphi^{-1}(y)}$.

Note that in particular, $\varphi\left(G_{x} \cdot x\right)=H_{\varphi(x)} \cdot \varphi(x)$.

In analogy to [21, Theorem 1.2], we obtain

Theorem 2.7. Let $G \curvearrowright X$ and $H \curvearrowright Y$ be topologically free partial systems. Then the following are equivalent:

(i) $G \curvearrowright X$ and $H \curvearrowright Y$ are continuously orbit equivalent,

(ii) the transformation groupoids $G \ltimes X$ and $H \ltimes Y$ are isomorphic as topological groupoids,

(iii) there exists an isomorphism $\Phi: C_{0}(X) \rtimes_{r} G \stackrel{\cong}{\longrightarrow} C_{0}(Y) \rtimes_{r} H$ with $\Phi\left(C_{0}(X)\right)=C_{0}(Y)$.

Moreover, "(ii) $\Rightarrow$ (i)" holds in general (i.e., without the assumption of topological freeness).

Proof. The proof is completely analogous to the one of [21, Theorem 1.2]. Therefore, we refer the reader to [21] for details, and only mention the key ideas.

For "(i) $\Rightarrow$ (ii)", observe that $G \ltimes X \rightarrow H \ltimes Y,(g, x) \mapsto(a(g, x), \varphi(x))$ and $H \ltimes Y \rightarrow$ $G \ltimes X,(h, y) \mapsto\left(b(h, y), \varphi^{-1}(y)\right)$ are continuous homomorphisms of groupoids which are inverse to each other. Here $\varphi, a$ and $b$ are as in Definition 2.6. This uses topological freeness as in [21].

For "(ii) $\Rightarrow$ (i)", let $\chi: G \ltimes X \rightarrow H \ltimes Y$ be an isomorphism of topological groupoids. Set $\varphi:=\left.\chi\right|_{X}, a$ as the composition $\bigcup_{g \in G}\{g\} \times U_{g^{-1}} \rightarrow G \ltimes X \stackrel{\chi}{\longrightarrow} H \ltimes Y \rightarrow H$, and $b$ as the composition $\bigcup_{h \in H}\{h\} \times V_{h^{-1}} \rightarrow H \ltimes Y \stackrel{\chi^{-1}}{\longrightarrow} G \ltimes X \rightarrow G$. Then it is easy to check that $\varphi, a$ and $b$ satisfy the conditions in Definition 2.6. This does not use topological freeness. 
For "(ii) $\Leftrightarrow$ (iii)", observe that by Corollary 2.5 and [34, Theorem 5.2], $\left(C_{0}(X) \rtimes_{r} G, C_{0}(X)\right) \cong$ $\left(C_{r}^{*}(G \ltimes X), C_{0}(X)\right)$ and $\left(C_{0}(Y) \rtimes_{r} H, C_{0}(Y)\right) \cong\left(C_{r}^{*}(H \ltimes Y), C_{0}(Y)\right)$ are Cartan pairs, in the sense of [34]. Then apply [34, Proposition 4.13].

\section{EXAMPles: InVERSE SEMIGROUPS, GRAPHS, AND SUBSEMIGROUPS OF GROUPS}

3.1. Inverse semigroups. Let $S$ be an inverse semigroup and $E$ the semilattice of idempotents of $S$. We set $S^{\times}=S \backslash\{0\}$ and $E^{\times}=E \backslash\{0\}$, i.e., we take away zero if $S$ has a zero. Assume that $\sigma$ is a partial homomorphism from $S$ to a group $G$ which is idempotent pure, i.e., $\sigma$ is a map $S^{\times} \rightarrow G$ such that $\sigma(s t)=\sigma(s) \sigma(t)$ for all $s, t \in S^{\times}$with $s t \in S^{\times}$, and that $\sigma^{-1}(e)=E^{\times}$.

In this situation, we describe a partial action $G \curvearrowright C^{*}(E)$ such that the (left) reduced $\mathrm{C}^{*}$-algebra $C_{\lambda}^{*}(S)$ of $S$ is canonically isomorphic to $C^{*}(E) \rtimes_{r} G$. This is the analogue of [29, Theorem 5.2], but without the assumption that $G$ is the universal group. Our observation gives us more freedom in the choice of idempotent pure partial homomorphisms. This will lead to a very simple criterion for nuclearity of inverse semigroup $\mathrm{C}^{*}$-algebras.

First, recall the definition of $C_{\lambda}^{*}(S)$ : For $s \in S$, let $\lambda_{s}: \ell^{2} S^{\times} \rightarrow \ell^{2} S^{\times}, \delta_{x} \mapsto \delta_{s x}$ if $s^{*} s \geq x x^{*}$ and $\delta_{x} \mapsto 0$ else. The reduced $\mathrm{C}^{*}$-algebra $C_{\lambda}^{*}(S)$ of $S$ is the sub-C*-algebra of $\mathcal{L}\left(\ell^{2} S^{\times}\right)$generated by $\left\{\lambda_{s}: s \in S\right\}$. Note that by construction, $\lambda_{0}=0$ if $0 \in S$. This is why we work with $\ell^{2} S^{\times}$ instead of $\ell^{2} S$. Let $C^{*}(E):=C^{*}\left(\left\{\lambda_{e}: e \in E\right\}\right) \subseteq C_{\lambda}^{*}(S)$. In the following, we write $e$ for $\lambda_{e} \in C^{*}(E)$. The full inverse semigroup $\mathrm{C}^{*}$-algebra $C^{*}(S)$ of an inverse semigroup $S$ is the universal $\mathrm{C}^{*}$-algebra for ${ }^{*}$-representations of $S$ by partial isometries. We mod out 0 if $S$ has a zero, as in [31].

Now let us describe the partial action $G \curvearrowright C^{*}(E)$. For $g \in G$, let $D_{g}$ be the sub-C*algebra (actually ideal) of $C^{*}(E)$ given by $D_{g^{-1}}=\overline{\operatorname{span}}\left(\left\{s^{*} s: s \in S^{\times}, \sigma(s)=g\right\}\right)$. As $\sigma$ is idempotent pure, we have $D_{e}=C^{*}(E)$. For every $g \in G$, we have a $\mathrm{C}^{*}$-isomorphism $\alpha_{g}^{*}: D_{g^{-1}} \rightarrow D_{g}, s^{*} s \mapsto s s^{*}$. The corresponding dual action is given as follows: Let $\widehat{E}=$ $\operatorname{Spec}\left(C^{*}(E)\right)$, and for every $g \in G$, set $U_{g}=\operatorname{Spec}\left(D_{g}\right) \subseteq \widehat{E}$. It is easy to see that $U_{g^{-1}}=$ $\left\{\chi \in \widehat{E}: \chi\left(s^{*} s\right)=1\right.$ for some $s \in S^{\times}$with $\left.\sigma(s)=g\right\} . \quad \alpha_{g}: U_{g^{-1}} \rightarrow U_{g}$ is given by $\alpha_{g}(\chi)=$ $\chi \circ \alpha_{g^{-1}}^{*}$. Given $\chi \in U_{g^{-1}}$ and $s \in S^{\times}$with $\sigma(s)=g$ and $\chi\left(s^{*} s\right)=1$, we have $\alpha_{g}(\chi)(e)=\chi\left(s^{*} e s\right)$.

The same proof as in [29] gives an explicit identification of the universal groupoid of $S$, in the sense of $[32, \S 4.3]$, but restricted to $\widehat{E}$, with the partial transformation groupoid $G \ltimes \widehat{E}$. We have to restrict to $\widehat{E}$ because in case $0 \in S$, the unit space of the universal groupoid in [32, $\S 4.3]$ is $\widehat{E} \sqcup\left\{\chi_{0}\right\}$, where $\chi_{0}$ corresponds to the character sending every idempotent in $E$ to 1 , even 0 . As a consequence of this identification of groupoids, we obtain

Proposition 3.1. We have $C_{\lambda}^{*}(S) \cong C_{r}^{*}(G \ltimes \widehat{E})$ and $C^{*}(S) \cong C^{*}(G \ltimes \widehat{E})$, and there are isomorphisms $C_{\lambda}^{*}(S) \rightarrow C^{*}(E) \rtimes_{r} G, \lambda_{s} \mapsto\left(s s^{*}\right) \delta_{\sigma(s)}$ and $C^{*}(S) \rightarrow C^{*}(E) \rtimes G, s \mapsto\left(s s^{*}\right) \delta_{\sigma(s)}$.

In many situations, one is interested not only in the reduced $\mathrm{C}^{*}$-algebra of the inverse semigroup, but also in its boundary quotient. Let us describe the partial system corresponding to this quotient. Given a semilattice $E$, let $\widehat{E}_{\max }$ be the subset of $\widehat{E}$ consisting of those $\chi \in \widehat{E}$ 
such that $\{e \in E: \chi(e)=1\}$ is maximal. We then set $\partial \widehat{E}:=\widehat{E}_{\max } \subseteq \widehat{E}$. Now let $E$ be the semilattice of idempotents in an inverse semigroup $S$. As $\partial \widehat{E} \subseteq \widehat{E}$ is closed, we obtain a short exact sequence $0 \rightarrow I \rightarrow C_{0}(\widehat{E}) \rightarrow C_{0}(\partial \widehat{E}) \rightarrow 0$. Viewing $I$ as a subset of $C_{\lambda}^{*}(S)$, we form the ideal $\langle I\rangle$ of $C_{\lambda}^{*}(S)$ generated by $I$. The reduced version of the boundary quotient in Exel's sense (see $[8,9,10,12])$ is given by $\partial C_{\lambda}^{*}(S):=C_{\lambda}^{*}(S) /\langle I\rangle$. In the particular case of inverse semigroups arising from right LCM semigroups, boundary quotients have been studied in [37].

Lemma 3.2. Let $S$ be an inverse semigroup as above, with an idempotent pure partial homomorphism $\sigma$ from $S$ to a group $G$, with semilattice of idempotents $\widehat{E}$ and partial system $G \curvearrowright \widehat{E}$. Then $\partial \widehat{E}$ is G-invariant.

Proof. Let us first show that for every $g \in G, g .\left(U_{g^{-1}} \cap \widehat{E}_{\max }\right) \subseteq U_{g} \cap \widehat{E}_{\text {max }}$. Take $\chi \in \widehat{E}_{\text {max }}$ with $\chi\left(s^{*} s\right)=1$ for some $s \in S$ with $\sigma(s)=g$. Then $g \cdot \chi(e)=\chi\left(s^{*} e s\right)$. Assume that $g \cdot \chi \notin \widehat{E}_{\max }$. This means that there is $\psi \in \widehat{E}_{\max }$ such that $\psi(e)=1$ for all $e \in E$ with $g \cdot \chi(e)=1$, and there exists $f \in E$ with $\psi(f)=1$ but $g \cdot \chi(f)=\chi\left(s^{*} f s\right)=0$. Then $\psi \in U_{g}$ since $g \cdot \chi\left(s s^{*}\right)=1$, which implies $\psi\left(s s^{*}\right)=1$. Consider $g^{-1} . \psi$ given by $g^{-1} \cdot \psi(e)=\psi\left(s e s^{*}\right)$. Then for every $e \in E$, $\chi(e)=1$ implies $\chi\left(s^{*}\right.$ ses $\left.^{*} s\right)=1$, hence $\chi\left(s^{*}\left(\right.\right.$ ses $\left.\left.^{*}\right) s\right)=1$, so that $g^{-1} \cdot \psi(e)=\psi\left(\right.$ ses $\left.s^{*}\right)=1$. But $\chi\left(s^{*} f s\right)=0$ and $g^{-1} \cdot \psi\left(s^{*} f s\right)=\psi\left(s s^{*} f s s^{*}\right)=\psi(f)=1$. This contradicts $\chi \in \widehat{E}_{\max }$. Hence $g .\left(U_{g^{-1}} \cap \widehat{E}_{\max }\right) \subseteq U_{g} \cap \widehat{E}_{\max }$. To see that $g .\left(U_{g^{-1}} \cap \partial \widehat{E}\right) \subseteq U_{g} \cap \partial \widehat{E}$, let $\chi \in U_{g^{-1}} \cap \partial \widehat{E}$ and choose a net $\left(\chi_{i}\right)_{i}$ in $\widehat{E}_{\max }$ with $\lim _{i} \chi_{i}=\chi$. As $U_{g^{-1}}$ is open, we may assume that all the $\chi_{i}$ lie in $U_{g^{-1}}$. Then $g \cdot \chi_{i} \in \widehat{E}_{\max }$, and $\lim _{i} g \cdot \chi_{i}=g \cdot \chi$. This implies $g \cdot \chi \in \partial \widehat{E}$.

Lemma 3.3. Let $S$ be an inverse semigroup with an idempotent pure partial homomorphism $\sigma$ from $S$ to an exact group $G$. Then the identification $C_{\lambda}^{*}(S) \cong C_{0}(\widehat{E}) \rtimes_{r} G$ from Proposition 3.1 identifies $\partial C_{\lambda}^{*}(S)$ with $C_{0}(\partial \widehat{E}) \rtimes_{r} G$.

Proof. It is easy to see that under the identification $C_{\lambda}^{*}(S) \cong C_{0}(\widehat{E}) \rtimes_{r} G$ from Proposition 3.1, $\langle I\rangle$ corresponds to $I \rtimes_{r} G$. Since $G$ is exact, we obtain a short exact sequence $0 \rightarrow I \rtimes_{r} G \rightarrow$ $C_{\lambda}^{*}(S) \rightarrow \partial C_{\lambda}^{*}(S) \rightarrow 0$ by $[11$, Theorem 22.9].

Corollary 3.4. Let $S$ be an inverse semigroup with an idempotent pure partial homomorphism $\sigma$ from $S$ to a group $G$. If $G$ is amenable, then both $C_{\lambda}^{*}(S)$ and $\partial C_{\lambda}^{*}(S)$ are nuclear. If $G$ is exact, then both $C_{\lambda}^{*}(S)$ and $\partial C_{\lambda}^{*}(S)$ are exact.

Proof. By Proposition 3.1 and Lemma 3.3, both $C_{\lambda}^{*}(S)$ and $\partial C_{\lambda}^{*}(S)$ can be described as reduced partial crossed products by $G$, hence as reduced $\mathrm{C}^{*}$-algebras of Fell bundles over $G$. Therefore, our claims follow from [11, Theorem 20.7, Theorem 25.10 and Theorem 25.12].

3.2. Graphs. Let $\mathcal{E}=\left(\mathcal{E}^{0}, \mathcal{E}^{1}, r, s\right)$ be a (countable) graph with vertices $\mathcal{E}^{0}$, edges $\mathcal{E}^{1}$ and range and source maps $r, s: \mathcal{E}^{1} \rightarrow \mathcal{E}^{0}$. Let $\mathcal{E}^{*}$ be the set of finite paths in $\mathcal{E}$, and let $l(\mu)$ denote the length of a path $\mu \in \mathcal{E}^{*}$. The graph inverse semigroup $S_{\mathcal{E}}$ is given by $S_{\mathcal{E}}=$ $\left\{(\mu, \nu) \in \mathcal{E}^{*} \times \mathcal{E}^{*}: s(\mu)=s(\nu)\right\} \cup\{0\}$, where $(\mu, \nu)^{*}=(\nu, \mu)$ and

$$
(\mu, \nu)(\zeta, \eta)= \begin{cases}\left(\mu, \nu^{\prime} \eta\right) & \text { if } \nu=\zeta \nu^{\prime} \\ \left(\mu \zeta^{\prime}, \eta\right) & \text { if } \zeta=\nu \zeta^{\prime} \\ 0 & \text { else. }\end{cases}
$$


The semilattice $E_{\mathcal{E}}$ of idempotents of $S_{\mathcal{E}}$ is given by $\left\{(\mu, \mu) \in \mathcal{E}^{*} \times \mathcal{E}^{*}\right\} \cup\{0\}$, hence can be identified canonically with $\mathcal{E}^{*} \cup\{0\}$. Multiplication in $E_{\mathcal{E}}$ is given by

$$
\mu \cdot \nu= \begin{cases}\mu & \text { if } \mu=\nu \mu^{\prime}, \\ \nu & \text { if } \nu=\mu \nu^{\prime}, \\ 0 & \text { else. }\end{cases}
$$

Note that we write $\mu \nu$ for concatenation of paths and $\mu \cdot \nu$ for the product in $E_{\mathcal{E}}$.

It is easy to see that $C_{\lambda}^{*}\left(S_{\mathcal{E}}\right)$ is canonically isomorphic to the Toeplitz $\mathrm{C}^{*}$-algebra of $\mathcal{E}$, and that $\partial C_{\lambda}^{*}\left(S_{\mathcal{E}}\right)$ is canonically isomorphic to the graph $\mathrm{C}^{*}$-algebra of $\mathcal{E}$.

Remark 3.5. Note that we are using the convention that the partial isometry $s_{\mu}$ for $\mu \in \mathcal{E}^{1}$ has source projection $e_{s(\mu)}$ corresponding to the source of $\mu$, and range projection dominated by $e_{r(\mu)}$, the projection corresponding to the range of $\mu$. This is the same convention as in [38], but different from the one in $[3,16]$.

Let us construct an idempotent pure partial homomorphism on $S_{\mathcal{E}}$. Let $\mathbb{F}_{\mathcal{E}^{1}}$ be the free group generated by $\mathcal{E}^{1}$. We view $\mu \in \mathcal{E}^{*}$ with $l(\mu) \geq 1$ as elements in $\mathbb{F}_{\mathcal{E}^{1}}$ in a canonical way. Define $\sigma: S_{\mathcal{E}}^{\times} \rightarrow \mathbb{F}_{\mathcal{E}^{1}}$ by setting $\sigma(\mu, \nu)=\mu \nu^{-1}$ if $l(\mu), l(\nu) \geq 1, \sigma(\mu, \nu)=\mu$ if $l(\mu) \geq 1$ and $l(\nu)=0$, $\sigma(\mu, \nu)=\nu^{-1}$ if $l(\mu)=0$ and $l(\nu) \geq 1$, and $\sigma(\mu, \nu)=e$ if $l(\mu)=l(\nu)=0$. Here $e$ is the identity of $\mathbb{F}_{\mathcal{E}^{1}}$. It is easy to check that $\sigma$ is an idempotent pure partial homomorphism from $S_{\mathcal{E}}$ to $\mathbb{F}_{\mathcal{E}^{1}}$. Clearly, $\sigma$ is the universal one, in the sense of [29].

Remark 3.6. Let us show how a modification of $\sigma$ produces an easy argument for nuclearity of graph $\mathrm{C}^{*}$-algebras. We write $\mathbb{F}_{\mathcal{E}^{1}}^{+}$for the free semigroup generated by $\mathcal{E}^{1}$. Let $\mathbb{F}_{2}^{+}$and $\mathbb{F}_{2}$ be the free semigroup and the free group on two generators. Clearly, there is a semigroup embedding $\mathbb{F}_{\mathcal{E}^{1}}^{+} \hookrightarrow \mathbb{F}_{2}^{+}\left(\mathcal{E}^{1}\right.$ is countable by assumption). Moreover, by [17], we have an embedding $\mathbb{F}_{2}^{+} \hookrightarrow$ $\mathbb{F}_{2} / \mathbb{F}_{2}^{\prime \prime}$, where $\mathbb{F}_{2}^{\prime \prime}$ is the second commutator subgroup of $\mathbb{F}_{2}$. Hence we obtain an embedding $\mathbb{F}_{\mathcal{E}^{1}}^{+} \hookrightarrow \mathbb{F}_{2}^{+} \hookrightarrow \mathbb{F}_{2} / \mathbb{F}_{2}^{\prime \prime}$. By universal property of $\mathbb{F}_{\mathcal{E}^{1}}$, we obtain a homomorphism $\mathbb{F}_{\mathcal{E}^{1}} \rightarrow \mathbb{F}_{2} / \mathbb{F}_{2}^{\prime \prime}$, which has to factorize as $\mathbb{F}_{\mathcal{E}^{1}} \rightarrow \mathbb{F}_{\mathcal{E}^{1}} / \mathbb{F}_{\mathcal{E}^{1}}^{\prime \prime} \rightarrow \mathbb{F}_{2} / \mathbb{F}_{2}^{\prime \prime}$, such that the diagram

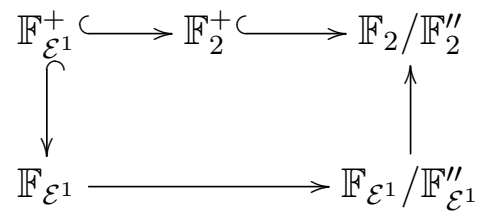

commutes. Thus, the canonical homomorphism $\mathbb{F}_{\mathcal{E}^{1}}^{+} \rightarrow \mathbb{F}_{\mathcal{E}^{1}} / \mathbb{F}_{\mathcal{E}^{1}}^{\prime \prime}$ is injective.

Now let $\sigma^{\prime \prime}$ be the composition $S_{\mathcal{E}}^{\times} \stackrel{\sigma}{\longrightarrow} \mathbb{F}_{\mathcal{E}^{1}} \rightarrow \mathbb{F}_{\mathcal{E}^{1}} / \mathbb{F}_{\mathcal{E}^{1}}^{\prime \prime} \sigma^{\prime \prime}$ is again a partial homomorphism, and $\sigma^{\prime \prime}$ is idempotent pure because $\mathbb{F}_{\mathcal{E}^{1}}^{+} \rightarrow \mathbb{F}_{\mathcal{E}^{1}} / \mathbb{F}_{\mathcal{E}^{1}}^{\prime \prime}$ is injective. Moreover, $\mathbb{F}_{\mathcal{E}^{1}} / \mathbb{F}_{\mathcal{E}^{1}}^{\prime \prime}$ is solvable, in particular amenable. Hence Corollary 3.4 implies that both the Toeplitz $\mathrm{C}^{*}$-algebra as well as the graph $\mathrm{C}^{*}$-algebra of $\mathcal{E}$ are nuclear.

Let us now come back to $\sigma: S_{\mathcal{E}}^{\times} \rightarrow \mathbb{F}_{\mathcal{E}^{1}}$, and describe the corresponding partial actions $\mathbb{F}_{\mathcal{E}^{1}} \curvearrowright \widehat{E_{\mathcal{E}}}$ and $\mathbb{F}_{\mathcal{E}^{1}} \curvearrowright \partial \mathcal{E}$, where $\partial \mathcal{E}:=\partial \widehat{E_{\mathcal{E}}}$. 
We start with $\mathbb{F}_{\mathcal{E}^{1}} \curvearrowright \widehat{E_{\mathcal{E}}}$. For $g \in \mathbb{F}_{\mathcal{E}^{1}}, U_{g^{-1}}$ is empty unless $g \in \operatorname{Im}(\sigma)$, i.e., $g=\alpha \beta^{-1}$ for some paths $\alpha, \beta \in\left(\mathcal{E}^{*} \backslash \mathcal{E}^{0}\right) \cup\{e\}$, and if $\alpha, \beta$ both lie in $\mathcal{E}^{*} \backslash \mathcal{E}^{0}$, then $s(\alpha)=s(\beta)$. For such $g, U_{g^{-1}}$ consists of those $\chi \in \widehat{E_{\mathcal{E}}}$ such that there exists $(\mu, \nu) \in S_{\mathcal{E}}^{\times}$with $\chi(\nu)=1$ and $\sigma(\mu, \nu)=\alpha \beta^{-1}$. For such $\chi$ with $(\mu, \nu)$ as above, $(g \cdot \chi)(\zeta)=\chi\left(\nu \zeta^{\prime}\right)$ if $\zeta=\mu \zeta^{\prime}$ and $(g \cdot \chi)(\zeta)=$ 0 otherwise. By Proposition 3.1, the Toeplitz $\mathrm{C}^{*}$-algebra of $\mathcal{E}$ is canonically isomorphic to $C_{\lambda}^{*}\left(S_{\mathcal{E}}\right) \cong C_{r}^{*}\left(\mathbb{F}_{\mathcal{E}^{1}} \ltimes \widehat{E_{\mathcal{E}}}\right) \cong C_{0}\left(\widehat{E_{\mathcal{E}}}\right) \rtimes_{r} \mathbb{F}_{\mathcal{E}^{1}}$

Following [38] (see also [13]), the partial system $\mathbb{F}_{\mathcal{E}^{1}} \curvearrowright \partial \mathcal{E}$ can be described explicitly as follows: As a set, we identify $\partial \mathcal{E}$ with $\mathcal{E}^{\infty} \cup\left\{\alpha \in \mathcal{E}^{*}: s(\alpha) \notin \mathcal{E}_{0}^{0}\right\}$, where $\mathcal{E}^{\infty}$ is the set of infinite paths in $\mathcal{E}$. To describe the topology, let $\mu \in \mathcal{E}^{*}$ and set

$$
\mathcal{Z}(\mu)=\left\{\nu \in \mathcal{E}^{*} \cup \mathcal{E}^{\infty}: \nu=\mu \nu^{\prime} \text { for some } \nu^{\prime} \in \mathcal{E}^{*} \cup \mathcal{E}^{\infty}\right\} .
$$

Given a finite subset $F$ of $r^{-1}(s(\mu))$, let $\mathcal{Z}(\mu \backslash F)=\mathcal{Z}(\mu) \backslash \bigcup_{\nu \in F} \mathcal{Z}(\mu \nu)$. Then

$$
\partial \mathcal{E} \cap \mathcal{Z}(\mu \backslash F), \mu \in \mathcal{E}^{*}, F \subseteq r^{-1}(s(\mu)) \text { finite }
$$

is a basis for the topology of $\partial \mathcal{E}$. The $\mathbb{F}_{\mathcal{E}^{1} \text {-action is just given by the restriction of the partial }}$ system $\mathbb{F}_{\mathcal{E}^{1}} \curvearrowright \widehat{E_{\mathcal{E}}}$, which has been described above. By Lemma 3.3, the graph $\mathrm{C}^{*}$-algebra of $\mathcal{E}$ is canonically isomorphic to $\partial C_{\lambda}^{*}\left(S_{\mathcal{E}}\right) \cong C_{r}^{*}\left(\mathbb{F}_{\mathcal{E}^{1}} \ltimes \partial \mathcal{E}\right) \cong C_{0}(\partial \mathcal{E}) \rtimes_{r} \mathbb{F}_{\mathcal{E}^{1}}$ (compare $[8$, Theorem 20.9]).

It is known (see [3, Proposition 2.3], and also Lemma 3.7) that $\mathbb{F}_{\mathcal{E}^{1}} \ltimes \partial \mathcal{E}$ is topologically principal, or equivalently (see Corollary 2.5), that $\mathbb{F}_{\mathcal{E}^{1}} \curvearrowright \partial \mathcal{E}$ is topologically free, if and only if $\mathcal{E}$ satisfies condition (L). Recall that $\mathcal{E}$ satisfies condition (L) if every loop has an entry, i.e., for every $\mu=\mu_{1} \cdots \mu_{n} \in \mathcal{E}^{*}$ with $\mu_{i} \in \mathcal{E}^{1}, n \geq 1$ and $s\left(\mu_{n}\right)=r\left(\mu_{1}\right)$, there is $\nu \in \mathcal{E}^{1}$ and $1 \leq i \leq n$ with $r(\nu)=r\left(\mu_{i}\right)$ and $\nu \neq \mu_{i}$.

Let us now relate our transformation groupoid to the groupoid attached to topological Markov shifts or graphs (see [25] and [3]), and our notion of continuous orbit equivalence for partial systems to continuous orbit equivalence for topological Markov shifts or graphs (see [25] and [3]). Once these relations are established, we will see that Theorem 2.7 applied to graphs generalizes [25, Theorem 2.3] and gives an alternative interpretation for [3, Theorem 5.1].

Let $\mathcal{E}$ be a graph as above. We compare the transformation groupoid $\mathbb{F}_{\mathcal{E}^{1}} \ltimes \partial \mathcal{E}$ with the groupoid $\mathcal{G}_{\mathcal{E}}$ from $[3, \S 2.3]$ (see $[25, \S 2.2]$ for the case of topological Markov shifts). The groupoid $\mathcal{G}_{\mathcal{E}}$ is given by $\mathcal{G}_{\mathcal{E}}=\left\{(\alpha, n, \beta) \in \partial \mathcal{E} \times \mathbb{Z} \times \partial \mathcal{E}: n=k-l\right.$ for $k, l \in \mathbb{Z}_{+}$and $\left.\sigma^{k}(\alpha)=\sigma^{l}(\beta)\right\}$. Here, we identify $\partial \mathcal{E}$ with $\mathcal{E}^{\infty} \cup\left\{\alpha \in \mathcal{E}^{*}: s(\alpha) \notin \mathcal{E}_{0}^{0}\right\}$ and define for $\mu=\mu_{1} \mu_{2} \mu_{3} \ldots$ in $\mathcal{E}^{\infty}$ with $l(\mu) \geq 2$ $\left(\mu_{i} \in \mathcal{E}^{1}\right) \sigma(\mu)=\mu_{2} \mu_{3} \ldots$, and $\sigma(\mu)=s(\mu)$ if $\mu \in \mathcal{E}^{1}$. For $\mu \in \mathcal{E}^{0}, \sigma$ is not defined. An equation like $\sigma^{k}(\alpha)=\sigma^{l}(\beta)$ always implicitly means that $\sigma^{k}(\alpha)$ and $\sigma^{l}(\beta)$ are defined, i.e., $l(\alpha) \geq k$ and $l(\beta) \geq l$. Moreover, we write $\mathbb{Z}_{+}=\{z \in \mathbb{Z}: z \geq 0\}$.

Lemma 3.7. $\mathbb{F}_{\mathcal{E}^{1}} \ltimes \partial \mathcal{E}$ and $\mathcal{G}_{\mathcal{E}}$ are isomorphic as topological groupoids.

Proof. It is easy to see that $\mathcal{G}_{\mathcal{E}} \rightarrow \mathbb{F}_{\mathcal{E}^{1}} \ltimes \partial \mathcal{E},(\lambda \nu, n, \mu \nu) \mapsto\left(\lambda \mu^{-1}, \mu \nu\right)$ and $\mathbb{F}_{\mathcal{E}^{1}} \ltimes \partial \mathcal{E} \rightarrow$ $\mathcal{G}_{\mathcal{E}},\left(\lambda \mu^{-1}, \mu \nu\right) \mapsto(\lambda \nu, l(\lambda)-l(\mu), \mu \nu)$ are mutually inverse (continuous) groupoid homomorphisms. (Note that these expressions for the maps only make sense for $l(\mu), l(\nu) \geq 1$. But there is an obvious way to define these maps for $l(\mu)=0$ or $l(\nu)=0$.) 
Let us also compare the notion of (continuous) orbit equivalence for graphs introduced in [3, Definition 3.1] (see also [25, § 2.1] for the case of topological Markov shifts) with continuous orbit equivalence of the corresponding partial systems. Let $\mathcal{E}$ and $\mathcal{F}$ be two graphs, with $\sigma_{\mathcal{E}}$ and $\sigma_{\mathcal{F}}$ as above, and partial systems $\mathbb{F}_{\mathcal{E}^{1}} \curvearrowright \partial \mathcal{E}$ and $\mathbb{F}_{\mathcal{F}^{1}} \curvearrowright \partial \mathcal{F}$. $\mathcal{E}$ and $\mathcal{F}$ are (continuously) orbit equivalent in the sense of [3, Definition 3.1] if there exists a homeomorphism $\varphi: \partial \mathcal{E} \rightarrow \partial \mathcal{F}$ together with continuous maps $k, l: \partial \mathcal{E} \rightarrow \mathbb{Z}_{+}$and $k^{\prime}, l^{\prime}: \partial \mathcal{F} \rightarrow \mathbb{Z}_{+}$such that

$$
\begin{aligned}
\sigma_{\mathcal{F}}^{k(\zeta)}\left(\varphi\left(\sigma_{\mathcal{E}}(\zeta)\right)\right) & =\sigma_{\mathcal{F}}^{l(\zeta)}(\varphi(\zeta)) \text { for all } \zeta \in \partial \mathcal{E}, \\
\sigma_{\mathcal{E}}^{k^{\prime}(\eta)}\left(\varphi^{-1}\left(\sigma_{\mathcal{F}}(\eta)\right)\right) & =\sigma_{\mathcal{E}}^{l^{\prime}(\eta)}\left(\varphi^{-1}(\eta)\right) \text { for all } \eta \in \partial \mathcal{F} .
\end{aligned}
$$

Lemma 3.8. If $\mathbb{F}_{\mathcal{E}^{1}} \curvearrowright \partial \mathcal{E}$ and $\mathbb{F}_{\mathcal{F}^{1}} \curvearrowright \partial \mathcal{F}$ are continuously orbit equivalent, then $\mathcal{E}$ and $\mathcal{F}$ are orbit equivalent.

If $\mathcal{E}$ and $\mathcal{F}$ satisfy condition $(L)$, then the converse holds, i.e., if $\mathcal{E}$ and $\mathcal{F}$ are orbit equivalent, then $\mathbb{F}_{\mathcal{E}^{1}} \curvearrowright \partial \mathcal{E}$ and $\mathbb{F}_{\mathcal{F}^{1}} \curvearrowright \partial \mathcal{F}$ are continuously orbit equivalent.

Proof. Assume that $\mathbb{F}_{\mathcal{E}^{1}} \curvearrowright \partial \mathcal{E}$ and $\mathbb{F}_{\mathcal{F}^{1}} \curvearrowright \partial \mathcal{F}$ are continuously orbit equivalent via $\varphi: \partial \mathcal{E} \cong \partial \mathcal{F}$ and continuous maps $a, b$ as in Definition 2.6. As $\partial \mathcal{E}=\left(\bigsqcup_{\zeta \in \mathcal{E}^{1}} \zeta \partial \mathcal{E}\right) \sqcup \mathcal{E}^{0} \backslash \mathcal{E}_{0}^{0}$, it suffices to define $k$ and $l$ on $\bigsqcup_{\zeta \in \mathcal{E}^{1}} \zeta \partial \mathcal{E}$ (on the remaining part, just set $k$ and $l$ to be 0 ). For $\zeta \in \mathcal{E}^{1}$ and $\zeta \xi \in \zeta \partial \mathcal{E}$, we know that $a\left(\zeta^{-1}, \zeta \xi\right)$ has the reduced form $\lambda \kappa^{-1}$, so that we can define $k$ and $l$ on $\zeta \partial \mathcal{E}$ by setting $k(\zeta \xi):=l(\lambda)$ and $l(\zeta \xi):=l(\kappa)$. These maps $k$ and $l$ are obviously locally constant, hence continuous. Moreover, we know that $\varphi\left(\zeta^{-1} \cdot(\zeta \xi)\right)=\left(\lambda \kappa^{-1}\right) \cdot \varphi(\zeta \xi)$, which means that there exists $\omega \in \partial \mathcal{F}$ with $\varphi(\zeta \xi)=\kappa \omega$ and $\varphi(\xi)=\varphi\left(\zeta^{-1} \cdot(\zeta \xi)\right)=\lambda \omega$. Therefore, $\sigma_{\mathcal{F}}^{l(\lambda)}\left(\varphi\left(\sigma_{\mathcal{E}}(\zeta \xi)\right)\right)=\sigma_{\mathcal{F}}^{l(\lambda)}(\varphi(\xi))=\sigma_{\mathcal{F}}^{l(\lambda)}(\lambda \omega)=\omega=\sigma_{\mathcal{F}}^{l(\kappa)}(\kappa \omega)=\sigma_{\mathcal{F}}^{l(\kappa)}(\varphi(\zeta \xi))$. Thus, (7) holds. $k^{\prime}$ and $l^{\prime}$ are defined in a similar way, using $b$.

Now assume that $\mathcal{E}$ and $\mathcal{F}$ satisfy condition $(\mathrm{L})$, and suppose conversely that $\mathcal{E}$ and $\mathcal{F}$ are orbit equivalent. Because of condition (L), our partial systems $\mathbb{F}_{\mathcal{E}^{1}} \curvearrowright \partial \mathcal{E}$ and $\mathbb{F}_{\mathcal{F}^{1}} \curvearrowright \partial \mathcal{F}$ are topologically free. Therefore, to prove that they are continuously orbit equivalent, all we have to show is that for every $g \in \mathbb{F}_{\mathcal{E}^{1}}$ and $x \in U_{g^{-1}}$, there exists an open neighbourhood $U$ of $x$ and $h \in \mathbb{F}_{\mathcal{F}^{1}}$ such that $\varphi(g . \bar{x})=h . \varphi(\bar{x})$ for all $\bar{x} \in U$, and analogously for $\varphi^{-1}$. In our case, since $\mathbb{F}_{\mathcal{E}^{1}}$ is generated by $\mathcal{E}^{1}$, it suffices to consider $g \in\left(\mathcal{E}^{1}\right)^{-1}$. Take $\zeta \in \mathcal{E}^{1}$ and $x \in \zeta \partial \mathcal{E}$. Choose $\lambda, \kappa \in \mathcal{F}^{*}$ with $l(\lambda) \geq k(x), l(\kappa) \geq l(x)$, such that $\varphi(x) \in \kappa \partial \mathcal{F}$ and $\varphi\left(\zeta^{-1} \cdot x\right) \in \lambda \partial \mathcal{F}$, and for all $\zeta \xi \in \zeta \partial \mathcal{E}$ with $\varphi(\zeta \xi) \in \kappa \partial \mathcal{F}$ and $\varphi(\xi)=\varphi\left(\zeta^{-1} .(\zeta \xi)\right) \in \lambda \partial \mathcal{F}$, we have $k(\zeta \xi)=k(x)$ and $l(\zeta \xi)=l(x)$. Such $\lambda$ and $\kappa$ exist because $k$ and $l$ are continuous, hence locally constant. Set $U:=\{\zeta \xi \in \zeta \partial \mathcal{E}: \varphi(\zeta \xi) \in \kappa \partial \mathcal{F}$ and $\varphi(\xi) \in \lambda \partial \mathcal{F}\} . U$ is obviously an open neighbourhood of $x$. Set $k:=k(x)$ and $l:=l(x)$. For all $\zeta \xi \in U$, we have $\sigma_{\mathcal{F}}^{k}(\varphi(\xi))=\sigma_{\mathcal{F}}^{k}\left(\varphi\left(\sigma_{\mathcal{E}}(\zeta \xi)\right)\right)=\sigma_{\mathcal{F}}^{l}(\varphi(\zeta \xi))$. Thus, when we write $\lambda=\lambda^{\prime} \lambda^{\prime \prime}$ with $l\left(\lambda^{\prime}\right)=k$ and $\kappa=\kappa^{\prime} \kappa^{\prime \prime}$ with $l\left(\kappa^{\prime}\right)=l$, we even know that $\left(\lambda^{\prime}\right)^{-1} \cdot \varphi(\xi)=\sigma_{\mathcal{F}}^{k}(\varphi(\xi))=\sigma_{\mathcal{F}}^{l}(\varphi(\zeta \xi))=\left(\kappa^{\prime}\right)^{-1} \cdot \varphi(\zeta \xi)$, and hence $\varphi\left(\zeta^{-1} \cdot(\zeta \xi)\right)=\left(\lambda^{\prime}\left(\kappa^{\prime}\right)^{-1}\right) \cdot \varphi(\zeta \xi)$ for all $\zeta \xi \in U$. Thus $\varphi$ has the desired property. The proof for $\varphi^{-1}$ is analogous.

Lemma 3.7, Lemma 3.8 and Theorem 2.7 imply the following

Theorem 3.9. Let $\mathcal{E}$ and $\mathcal{F}$ be graphs. Consider the statements

a) $\mathbb{F}_{\mathcal{E}^{1}} \ltimes \partial \mathcal{E}$ and $\mathbb{F}_{\mathcal{F}^{1}} \ltimes \partial \mathcal{F}$ are isomorphic as topological groupoids,

b) $\mathcal{G}_{\mathcal{E}}$ and $\mathcal{G}_{\mathcal{F}}$ are isomorphic as topological groupoids, 
c) there exists an isomorphism $\Phi: C^{*}(\mathcal{E}) \stackrel{\cong}{\longrightarrow} C^{*}(\mathcal{F})$ with $\Phi\left(C^{*}\left(E_{\mathcal{E}}\right)\right)=C^{*}\left(E_{\mathcal{F}}\right)$,

d) $\mathbb{F}_{\mathcal{E}^{1}} \curvearrowright \partial \mathcal{E}$ and $\mathbb{F}_{\mathcal{F}^{1}} \curvearrowright \partial \mathcal{F}$ are continuously orbit equivalent,

e) $\mathcal{E}$ and $\mathcal{F}$ are orbit equivalent.

We always have a) $\Leftrightarrow b)$, a) $\Rightarrow$ c) as well as a) $\Rightarrow d) \Rightarrow e$ ). If $\mathcal{E}$ and $\mathcal{F}$ satisfy condition $(L)$, then all these statements are equivalent.

This generalizes [25, Theorem 2.3] and gives an alternative proof for the corresponding parts of [3, Theorem 5.1]. Note that in [3], it is proven that a), b) and c) are always equivalent.

3.3. Subsemigroups of groups. Let us discuss $\mathrm{C}^{*}$-algebras attached to subsemigroups of groups. Given a subsemigroup $P$ of a group $G$ (or any left cancellative semigroup), the left reduced semigroup $\mathrm{C}^{*}$-algebra $C_{\lambda}^{*}(P)$ of $P$ is defined as the sub-C*-algebra of $\mathcal{L}\left(\ell^{2} P\right)$ generated by the left multiplication operators $V_{p}: \ell^{2} P \rightarrow \ell^{2} P, \delta_{x} \mapsto \delta_{p x}$. Here $\delta_{x}$ is the delta function in $x \in P$, and these $\delta_{x}$ form the canonical orthonormal basis of $\ell^{2} P$. The canonical commutative subalgebra $D_{\lambda}(P)$ is given by $D_{\lambda}(P)=C_{\lambda}^{*}(P) \cap \ell^{\infty}(P)$. Here is an alternative description of $D_{\lambda}(P)$ : Let $I_{V}$ be the inverse semigroup of partial isometries on $\ell^{2} P$ generated by $V_{p}$, $p \in P$, i.e., $I_{V}=\left\{V_{p_{1}}^{*} V_{q_{1}} \cdots V_{p_{n}}^{*} V_{q_{n}}: n \in \mathbb{N}, p_{i}, q_{i} \in P\right\}$. We can define a partial homomorphism $\sigma: I_{V}^{\times} \rightarrow G, V_{p_{1}}^{*} V_{q_{1}} \cdots V_{p_{n}}^{*} V_{q_{n}} \mapsto p_{1}^{-1} q_{1} \cdots p_{n}^{-1} q_{n}$. To see that $\sigma$ is well-defined, note that every $V \in I_{V}$ has the property that there exists $g \in G$ such that for every $x \in P$, either $V \delta_{x}=0$ or $V \delta_{x}=\delta_{g x}$. And $\sigma$ is defined in such a way that $\sigma(V)=g$. Now the closed linear span $\overline{\operatorname{span}}\left(\sigma^{-1}(e)\right)$ of $\sigma^{-1}(e)$, where $e$ is the identity of $G$, coincides with $D_{\lambda}(P)$. All this is explained in $[18,19]$ (see [18, Remark 3.12] for the alternative description of $D_{\lambda}(P)$ ).

Let us now describe the canonical partial action $G \curvearrowright D_{\lambda}(P)$. We first describe the dual action $\alpha^{*}$. For $g \in G$, let $D_{g^{-1}}:=\overline{\operatorname{span}}\left(\left\{V^{*} V: V \in I_{V}^{\times}, \sigma(V)=g\right\}\right)$. $D_{g^{-1}}$ is an ideal of $D_{\lambda}(P)$, and it follows from the alternative description of $D_{\lambda}(P)$ that $D_{e}=D_{\lambda}(P)$. We then define $\alpha_{g}^{*}$ as $\alpha_{g}^{*}: D_{g^{-1}} \rightarrow D_{g}, V^{*} V \rightarrow V V^{*}$ for $V \in I_{V}^{\times}$with $\sigma(V)=g$. This is well-defined: If we view $\ell^{2} P$ as a subspace $\ell^{2} G$ and let $\lambda$ be the left regular representation of $G$, then every $V \in I_{V}^{\times}$with $\sigma(V)=g$ satisfies $V=\lambda_{g} V^{*} V$. Therefore, $V V^{*}=\lambda_{g} V^{*} V \lambda_{g}^{*}$. This shows that $\alpha_{g}^{*}$ is just conjugation with the unitary $\lambda_{g}$. This also explains why $\alpha_{g}^{*}$ is an isomorphism. Of course, we can also describe the dual action $\alpha$. Set $\Omega_{P}:=\operatorname{Spec}\left(D_{\lambda}(P)\right)$ as the spectrum of the commutative $\mathrm{C}^{*}$-algebra $D_{\lambda}(P)$, and for every $g \in G$, let $U_{g^{-1}}:=\widehat{D_{g^{-1}}}$. Obviously, $U_{g^{-1}}=$ $\left\{\chi \in \Omega_{P}: \chi\left(V^{*} V\right)=1\right.$ for some $V \in I_{V}^{\times}$with $\left.\sigma(V)=g\right\}$. Define $\alpha_{g}$ by $\alpha_{g}(\chi):=\chi \circ \alpha_{g^{-1}}^{*}$.

Proposition 3.10. There is a canonical isomorphism $C_{\lambda}^{*}(P) \cong D_{\lambda}(P) \rtimes_{r} G$ determined by $V_{p} \mapsto V_{p} V_{p}^{*} \delta_{p}$. Here we form the partial crossed product for the partial action $G \curvearrowright D_{\lambda}(P)$ defined above, and $\delta_{g}$ denote the canonical partial isometries in $D_{\lambda}(P) \rtimes_{r} G$.

Proof. Our strategy is to describe both $C_{\lambda}^{*}(P)$ and $D_{\lambda}(P) \rtimes_{r} G$ as reduced (cross sectional) algebras of Fell bundles, and then to identify the underlying Fell bundles.

Let us start with $C_{\lambda}^{*}(P)$. We have already defined $I_{V}$ and $\sigma$. Now we set $B_{g}:=\overline{\operatorname{span}}\left(\sigma^{-1}(g)\right)$ for every $g \in G$. We want to see that $\left(B_{g}\right)_{g \in G}$ is a grading for $C_{\lambda}^{*}(P)$, in the sense of [7, Definition 3.1]. Conditions (i) and (ii) are obviously satisfied. For (iii), we use the faithful conditional expectation $\mathcal{E}: C_{\lambda}^{*}(P) \rightarrow D_{\lambda}(P)=B_{e}$ from $[18, \S 3.1]$. Given a finite sum $x=$ 
$\sum_{g} x_{g} \in C_{\lambda}^{*}(P)$ of elements $x_{g} \in B_{g}$ such that $x=0$, we conclude that $0=x^{*} x=\sum_{g, h} x_{g}^{*} x_{h}$, and hence $0=\mathcal{E}\left(x^{*} x\right)=\sum_{g} x_{g}^{*} x_{g}$ (here we used that $\left.\mathcal{E}\right|_{B_{g}}=0$ if $g \neq e$ ). This implies that $x_{g}=0$ for all $g$. Therefore, the subspaces $B_{g}$ are independent. It is clear that the linear span of all the $B_{g}$ is dense in $C_{\lambda}^{*}(P)$. This proves (iii). If we let $\mathcal{B}$ be the Fell bundle given by $\left(B_{g}\right)_{g \in G}$, then [7, Proposition 3.7] implies $C_{\lambda}^{*}(P) \cong C_{r}^{*}(\mathcal{B})$ because $\mathcal{E}: C_{\lambda}^{*}(P) \rightarrow D_{\lambda}(P)=B_{e}$ is a faithful conditional expectation satisfying $\left.\mathcal{E}\right|_{B_{e}}=\operatorname{id}_{B_{e}}$ and $\left.\mathcal{E}\right|_{B_{g}}=0$ if $g \neq e$.

Let us also describe $D_{\lambda}(P) \rtimes_{r} G$ as a reduced algebra of a Fell bundle. We denote by $W_{g}$ the partial isometry in $D_{\lambda}(P) \rtimes_{r} G$ corresponding to $g \in G$, and we set $B_{g}^{\prime}:=D_{g} W_{g}$. Recall that we defined $D_{g^{-1}}=\overline{\operatorname{span}}\left(\left\{V^{*} V: V \in I_{V}^{\times}, \sigma(V)=g\right\}\right)$ earlier on. It is easy to check that $\left(B_{g}^{\prime}\right)_{g \in G}$ satisfy (i), (ii) and (iii) in [7, Definition 3.1]. Moreover, $B_{e}^{\prime}=D_{e}=D_{\lambda}(P)$, and it follows immediately from the construction of the reduced partial crossed product that there is a faithful conditional expectation $D_{\lambda}(P) \rtimes_{r} G \rightarrow D_{\lambda}(P)=B_{e}^{\prime}$ which is identity on $B_{e}^{\prime}$ and 0 on $B_{g}^{\prime}$ for $g \neq e$. Hence if we let $\mathcal{B}^{\prime}$ be the Fell bundle given by $\left(B_{g}^{\prime}\right)_{g \in G}$, then [7, Proposition 3.7] implies $D_{\lambda}(P) \rtimes_{r} G \cong C_{r}^{*}\left(\mathcal{B}^{\prime}\right)$.

To identify $C_{\lambda}^{*}(P)$ and $D_{\lambda}(P) \rtimes_{r} G$, it now remains to identify $\mathcal{B}$ with $\mathcal{B}^{\prime}$. We claim that the map $\operatorname{span}(\{V: \sigma(V)=g\}) \rightarrow \operatorname{span}\left(\left\{V V^{*} W_{g}: \sigma(V)=g\right\}\right), \sum_{i} \alpha_{i} V_{i} \mapsto \sum_{i} \alpha_{i} V_{i} V_{i}^{*} W_{g}$ is well-defined and extends to an isometric isomorphism $B_{g} \rightarrow B_{g}^{\prime}$, for all $g \in G$.

All we have to show is that our map is isometric. We have $\left\|\sum_{i} \alpha_{i} V_{i}\right\|^{2}=\left\|\sum_{i, j} \alpha_{i} \overline{\alpha_{j}} V_{i} V_{j}^{*}\right\|_{D_{\lambda}(P)}$ and $\left\|\sum_{i} \alpha_{i} V_{i} V_{i}^{*} W_{g}\right\|^{2}=\left\|\sum_{i, j} \alpha_{i} \overline{\alpha_{j}} V_{i} V_{i}^{*} V_{j} V_{j}^{*}\right\|_{D_{\lambda}(P)}$. Since $V_{i}=V_{i} V_{i}^{*} \lambda_{g}$ and $V_{j}^{*}=\lambda_{g^{-1}} V_{j} V_{j}^{*}$, we have $V_{i} V_{j}^{*}=V_{i} V_{i}^{*} \lambda_{g} \lambda_{g^{-1}} V_{j} V_{j}^{*}=V_{i} V_{i}^{*} V_{j} V_{j}^{*}$. Hence, indeed, $\left\|\sum_{i} \alpha_{i} V_{i}\right\|^{2}=\left\|\sum_{i} \alpha_{i} V_{i} V_{i}^{*} W_{g}\right\|^{2}$, and we are done.

All in all, we have proven that $C_{\lambda}^{*}(P) \cong C_{r}^{*}(\mathcal{B}) \cong C_{r}^{*}\left(\mathcal{B}^{\prime}\right) \cong D_{\lambda}(P) \rtimes_{r} G$.

Remark 3.11. A straightforward computation shows that actually, $V_{p} V_{p}^{*} \delta_{p}=\delta_{p}$ for all $p \in P$. Thus the isomorphism in the Proposition 3.10 is given by $V_{p} \mapsto \delta_{p}$ for all $p \in P$.

Our next goal is to write $C_{\lambda}^{*}(P)$ as a quotient of a $\mathrm{C}^{*}$-algebra of an inverse semigroup in a canonical way. Let $S:=I_{l}(P)$ be the inverse semigroup of partial bijections of $P$ generated by $p: P \stackrel{\cong}{\longrightarrow} P, x \mapsto p x(p \in P)$. The semilattice of idempotents $E$ of $S$ is given by the set of constructible ideals $\mathcal{J}=\left\{p_{1}^{-1} q_{1} \cdots p_{n}^{-1} q_{n} P: p_{i}, q_{i} \in P\right\}$ (see [18, $\left.\S 2.1\right]$ ). It is easy to see that $S$ is canonically isomorphic to the inverse semigroup $I_{V}$ constructed above. The homomorphism $I_{V}^{\times} \rightarrow G$ from above yields an idempotent pure partial homomorphism from $S$ to $G$ such that for every $s \in S^{\times}, s(x)=\sigma(s) \cdot x$ if $x \in \operatorname{dom}(s)$.

As explained in [31, Corollary 3.2.13], the isometry $\ell^{2} P \rightarrow \ell^{2} S^{\times}, \delta_{p} \mapsto \delta_{p}$ induces surjective homomorphisms $C^{*}(E) \rightarrow D_{\lambda}(P)$ and $C_{\lambda}^{*}(S) \rightarrow C_{\lambda}^{*}(P)$. The first surjection allows us to view $\Omega_{P}=\operatorname{Spec}\left(D_{\lambda}(P)\right)$ as a closed subspace of $\widehat{E}$. More precisely, $\chi \in \widehat{E}$ lies in $\Omega_{P}$ if for all constructible ideals $X, X_{1}, \ldots, X_{n}$ of $P$ with $X=\bigcup_{i=1}^{n} X_{i}, \chi(X)=1$ implies $\chi\left(X_{i}\right)=1$ for some $1 \leq i \leq n$.

The following lemma is easy to check: 
Lemma 3.12. $\Omega_{P}$ is a $G$-invariant subspace with respect to the canonical $G$-action on $\widehat{E}$. The corresponding partial system $G \curvearrowright \Omega_{P}$ coincides with $\alpha$, the dual action of $\alpha^{*}: G \curvearrowright D_{\lambda}(P)$ constructed before Proposition 3.10.

Moreover, the surjection $C_{\lambda}^{*}(S) \rightarrow C_{\lambda}^{*}(P)$ from [31, Corollary 3.2.13] corresponds to the obvious map $C(\widehat{E}) \rtimes_{r} G \rightarrow C\left(\Omega_{P}\right) \rtimes_{r} G$ under the identifications given by Proposition 3.1 and Proposition 3.10.

Here is a sufficient condition for topological freeness of $G \curvearrowright \Omega_{P}$ :

Proposition 3.13. If $P$ contains the identity $e \in G$, and if the group of units $P^{*}$ in $P$ is trivial, i.e., $P^{*}=\{e\}$, then $G \curvearrowright \Omega_{P}$ is topologically free.

Proof. For $p \in P$, let $\chi_{p} \in \widehat{E}$ be defined by $\chi_{p}(X)=1$ if and only if $p \in X$, for $X \in \mathcal{J}$. Obviously, $\chi_{p} \in \Omega_{P}$. It turns out that $\left\{\chi_{p}: p \in P\right\}$ is dense in $\Omega_{P}$. Basic open sets in $\Omega_{P}$ are of the form $U\left(X ; X_{1}, \ldots, X_{n}\right)=\left\{\chi \in \Omega_{P}: \chi(X)=1, \chi\left(X_{i}\right)=0\right.$ for all $\left.1 \leq i \leq n\right\}$. Here $X, X_{1}, \ldots, X_{n}$ are constructible ideals of $P$. Clearly, $U\left(X ; X_{1}, \ldots, X_{n}\right)$ is empty if $X=\bigcup_{i=1}^{n} X_{i}$. Thus, for a non-empty basic open set $U\left(X ; X_{1}, \ldots, X_{n}\right)$, we may choose $p \in X$ such that $p \notin \bigcup_{i=1}^{n} X_{i}$, and then $\chi_{p} \in U\left(X ; X_{1}, \ldots, X_{n}\right)$.

Let $p \in P$ and $g \in G$ satisfy $g \cdot \chi_{p}=\chi_{p}$. This equality only makes sense if $\chi_{p} \in U_{g^{-1}}$, i.e., there exists $Y \in \mathcal{J}$ with $Y \subseteq g^{-1} \cdot P$ and $p \in Y$. Then $g \cdot \chi_{p}(X)=\chi_{p}\left(g^{-1} \cdot(X \cap g \cdot Y)\right)$. So $g \cdot \chi_{p}(X)=1$ if and only if $p \in g^{-1} \cdot(X \cap g \cdot Y)$ if and only if $g p \in X \cap g \cdot Y$ if and only if $g p \in X$, while $\chi_{p}(X)=1$ if and only if $p \in P$. Hence $g \cdot \chi_{p}=\chi_{p}$ implies that for every $X \in \mathcal{J}, g p \in X$ if and only if $p \in X$. For $X=p P$, we obtain $g p \in p P$, and for $X=g p P$, we get $p \in g p P$. Hence there exist $x, y \in P$ with $g p=p x$ and $p=g p y$. So $p=g p y=p x y$ and $g p=p x=g p y x$. Thus $x y=y x=e$. Hence $x, y \in P^{*}$. Since $P^{*}=\{e\}$ by assumption, we must have $x=y=e$, and hence $g p=p$. This implies $g=e$. In other words, for every $e \neq g \in G, g \cdot \chi_{p} \neq \chi_{p}$ for all $p \in P$ such that $\chi_{p} \in U_{g^{-1}}$. Hence it follows that $\left\{\chi \in U_{g^{-1}}: g \cdot \chi \neq \chi\right\}$ contains $\left\{\chi_{p} \in U_{g^{-1}}: p \in P\right\}$, and the latter set is dense in $U_{g^{-1}}$ as $\left\{\chi_{p}: p \in P\right\}$ is dense in $\Omega_{P}$.

Coming back to the comparison of $C_{\lambda}^{*}(S)$ and $C_{\lambda}^{*}(P)$, it was shown in [31, Theorem 3.2.14] that the following are equivalent:

- the canonical map $C_{\lambda}^{*}(S) \rightarrow C_{\lambda}^{*}(P)$ is injective,

- the canonical map $C^{*}(E) \rightarrow D_{\lambda}(P)$ is injective,

- $\mathcal{J}$ is independent.

Recall that $\mathcal{J}$ is called independent if for all $X, X_{1}, \ldots, X_{n} \in \mathcal{J}, X=\bigcup_{i=1}^{n} X_{i}$ implies $X=X_{i}$ for some $1 \leq i \leq n$.

In view of $[18,19]$, it makes sense to view the full $\mathrm{C}^{*}$-algebra $C^{*}(S)$ of the inverse semigroup $S=I_{l}(P)$ as the full semigroup $\mathrm{C}^{*}$-algebra of $P$. Thus we set $C^{*}(P):=C^{*}(S)$, and let $\lambda: C^{*}(P) \rightarrow C_{\lambda}^{*}(P)$ be the composite $C^{*}(P)=C^{*}(S) \rightarrow C_{\lambda}^{*}(S) \rightarrow C_{\lambda}^{*}(P)$. Because of our descriptions as transformation groupoids (see Proposition 3.1), the following generalizations of [19, Theorem 6.1] are immediate.

Theorem 3.14. If $G \ltimes \widehat{E}$ or $G \ltimes \Omega_{P}$ is amenable, then $\lambda: C^{*}(P) \rightarrow C_{\lambda}^{*}(P)$ is an isomorphism if and only if $P$ is independent. 
Theorem 3.15. Consider the following statements:

(i) $C^{*}(P)$ is nuclear,

(ii) $C_{\lambda}^{*}(P)$ is nuclear,

(iii) $G \ltimes \Omega_{P}$ is amenable,

(iv) $\lambda: C^{*}(P) \rightarrow C_{\lambda}^{*}(P)$ is an isomorphism.

We always have (i) $\Rightarrow$ (ii) $\Leftrightarrow$ (iii). If $P$ is independent, then (iii) $\Rightarrow$ (iv) and (iii) $\Rightarrow$ (i), so that (i), (ii) and (iii) are equivalent.

It is an open problem whether all the items (i) to (iv) are equivalent for semigroups which are independent.

Corollary 3.16 (compare Proposition 3.46 in [31]). If $P$ is a subsemigroup of an amenable group $G$, then (i), (ii) and (iii) from Theorem 3.15 hold, and (iv) is true if and only if $P$ is independent.

Proof. By Proposition 3.1, both $C^{*}(P)$ and $C_{\lambda}^{*}(P)$ can be written as (full or reduced) partial crossed products by $G$, and hence as (full or reduced) $\mathrm{C}^{*}$-algebras of Fell bundles over $G$. Therefore, by [11, Theorem 20.7 and Theorem 25.10], $C^{*}(P)$ and $C_{\lambda}^{*}(P)$ are nuclear. (iii) holds because we know that (ii) $\Leftrightarrow$ (iii). Finally, our claim about (iv) follows from Theorem 3.14.

Now set $\partial \Omega_{P}:=\partial \widehat{E}$, where $E$ is the semilattice of idempotents of $S=I_{l}(P)$.

Lemma 3.17. We have $\partial \Omega_{P} \subseteq \Omega_{P}$.

Proof. Let $X, X_{1}, \ldots, X_{n} \in \mathcal{J}$ satisfy $X=\bigcup_{i=1}^{n} X_{i}$. Then for $\chi \in \widehat{E}_{\max }, \chi\left(X_{i}\right)=0$ implies that there exists $X_{i}^{\prime} \in \mathcal{J}$ with $\chi\left(X_{i}^{\prime}\right)=1$ and $X_{i} \cap X_{i}^{\prime}=\emptyset$. Thus if $\chi\left(X_{i}\right)=0$ for all $1 \leq i \leq n$, then let $X_{i}^{\prime}, 1 \leq i \leq n$ be as above. Then for $X^{\prime}=\bigcap_{i=1}^{n} X_{i}^{\prime}, \chi\left(X^{\prime}\right)=1$ and $X \cap X^{\prime}=\emptyset$. Thus $\chi(X)=0$. This shows $\widehat{E}_{\max } \subseteq \Omega_{P}$. As $\Omega_{P}$ is closed, we conclude that $\partial \widehat{E} \subseteq \Omega_{P}$.

As $\partial \Omega_{P}$ is $G$-invariant, the following makes sense.

Definition 3.18. The boundary quotient of $C_{\lambda}^{*}(P)$ is given by $\partial C_{\lambda}^{*}(P):=C\left(\partial \Omega_{P}\right) \rtimes_{r} G$.

By construction and because of Proposition 3.10, there is a canonical projection $C_{\lambda}^{*}(P) \rightarrow$ $\partial C_{\lambda}^{*}(P)$. Morever, if $P$ is a subsemigroup of an exact group $G$, then $\partial C_{\lambda}^{*}(P)$ is isomorphic to $\partial C_{\lambda}^{*}(S) \cong C\left(\partial \Omega_{P}\right) \rtimes_{r} G$. Let us now generalize the results in $[19, \S 7.3]$.

Lemma 3.19. $\partial \widehat{E}=\partial \Omega_{P}$ is the minimal non-empty closed $G$-invariant subspace of $\widehat{E}$.

Proof. Let $C \subseteq \widehat{E}$ be non-empty, closed and $G$-invariant. Let $\chi \in \widehat{E}_{\max }$ be arbitrary, and choose $X \in \mathcal{J}$ with $\chi(X)=1$. Choose $p \in X$ and $\chi \in C$. As $U_{p^{-1}}=\widehat{E}$, we can form $p \cdot \chi$, and we know that $p \cdot \chi \in C$. We have $p \cdot \chi(p P)=\chi(P)=1$, so that $p \cdot \chi(X)=1$ as $p \in X$ implies $p P \subseteq X\left(X\right.$ is a right ideal). Set $\chi_{X}:=p \cdot \chi$. Consider the net $\left(\chi_{X}\right)_{X}$ indexed by $X \in \mathcal{J}$ with $\chi(X)=1$, ordered by inclusion. Passing to a convergent subnet if necessary, we may assume that $\lim _{X} \chi_{X}$ exists. But it is clear because of $\chi \in \widehat{E}_{\max }$ that $\lim _{X} \chi_{X}=\chi$. As $\chi_{X} \in C$ for all $X$, we deduce that $\chi \in C$. Thus $\widehat{E}_{\max } \subseteq C$, and hence $\partial \widehat{E} \subseteq C$.

In particular, $\partial \Omega_{P}$ is the minimal non-empty closed $G$-invariant subspace of $\Omega_{P}$. Another immediate consequence is 
Corollary 3.20. The transformation groupoid $G \ltimes \partial \Omega_{P}$ is minimal.

To discuss topological freeness of $G \curvearrowright \partial \Omega_{P}$, let

$$
G_{0}=\left\{g \in G: X \cap g \cdot P \neq \emptyset \neq X \cap g^{-1} \cdot P \text { for all } \emptyset \neq X \in \mathcal{J}\right\},
$$

as in [19, § 7.3]. Clearly, $G_{0}=\left\{g \in G: p P \cap g \cdot P \neq \emptyset \neq p P \cap g^{-1} \cdot P\right.$ for all $\left.p \in P\right\}$. Furthermore, [19, Lemma 7.19] shows that $G_{0}$ is a subgroup of $G$.

Proposition 3.21. $G \curvearrowright \partial \Omega_{P}$ is topologically free if and only if $G_{0} \curvearrowright \partial \Omega_{P}$ is topologically free.

Proof. " $\Rightarrow$ " is clear. For " $\Leftarrow$ ", assume that $G_{0} \curvearrowright \partial \Omega_{P}$ is topologically free, and suppose that $G \curvearrowright \partial \Omega_{P}$ is not topologically free, i.e., there exists $g \in G$ and $U \subseteq U_{g^{-1}} \cap \partial \Omega_{P}$ such that $g \cdot \chi=\chi$ for all $\chi \in U$. As ${\widehat{E_{\max }}}=\partial \Omega_{P}$, we can find $\chi \in U_{g^{-1}} \cap \widehat{E}_{\max }$ with $g \cdot \chi=\chi$.

For every $X \in \mathcal{J}$ with $\chi(X)=1$, choose $x \in X$ and $\psi_{X} \in \widehat{E}_{\max }$ with $\psi_{X}(x P)=1$, so that $\psi_{X}(X)=1$. Consider the net $\left(\psi_{X}\right)_{X}$ indexed by $X \in \mathcal{J}$ with $\chi(X)=1$, ordered by inclusion. Passing to a convergent subnet if necessary, we may assume that $\lim _{X} \psi_{X}=\chi$. As $U$ is open, we may assume that $\psi_{X} \in U$ for all $X$. Then $\psi_{X}(x P)=1$ implies that $\psi_{X} \in U_{x} \cap U$.

Hence for sufficiently small $X \in \mathcal{J}$ with $\chi(X)=1$, there exists $x \in X$ such that $x^{-1}$. $\left(U_{x} \cap U\right)$ is a non-empty open subset of $\partial \Omega_{P}$. We conclude that $\left(x^{-1} g x\right) . \psi=\psi$ for all $\psi \in x^{-1}$. $\left(U_{x} \cap U\right)$. This implies that $x^{-1} g x \notin G_{0}$ as $G_{0} \curvearrowright \partial \Omega_{P}$ is topologically free. So there exists $p \in P$ with $p P \cap x^{-1} g x \cdot P=\emptyset$ or $p P \cap x^{-1} g^{-1} x \cdot P=\emptyset$. Let $\chi_{X} \in \widehat{E}_{\max }$ satisfy $\chi_{X}(x p P)=1$. If $p P \cap x^{-1} g x \cdot P=\emptyset$, then $x p P \cap g x \cdot P=\emptyset$, so that $x p P \cap g^{-1} x p \cdot P=\emptyset$. Hence $g \cdot \chi_{X} \neq \chi_{X}$ if $\chi_{X} \in U_{g^{-1}}$. If $p P \cap x^{-1} g^{-1} x \cdot P=\emptyset$, then $x p P \cap g^{-1} x \cdot P=\emptyset$, so that $x p P \cap g^{-1} x p \cdot P=\emptyset$. Again, $g \cdot \chi_{X} \neq \chi_{X}$ if $\chi_{X} \in U_{g^{-1}}$.

For every sufficiently small $X \in \mathcal{J}$ with $\chi(X)=1$, we can find $x \in X$ and $\chi_{X}$ as above. Hence we can consider the net $\left(\chi_{X}\right)_{X}$ as above, and assume after passing to a convergent subnet that $\lim _{X} \chi_{X}=\chi$. As $\chi \in U \subseteq U_{g^{-1}} \cap \partial \Omega_{P}$, it follows that $\chi_{X} \in U \subseteq U_{g^{-1}} \cap \partial \Omega_{P}$ for sufficiently small $X$. So we obtain $g \cdot \chi_{X} \neq \chi_{X}$, although $g$ acts trivially on $U$. This is a contradiction.

Corollary 3.22. If $G_{0} \curvearrowright \partial \Omega_{P}$ is topologically free, then $\partial C_{\lambda}^{*}(P)$ is simple.

Proof. This follows from Lemma 3.19, Proposition 3.21 and [33, Chapter II, Proposition 4.6].

Theorem 3.23. Let $P=\langle\mathcal{S}, \mathcal{R}\rangle^{+}$be a monoid given by a positive $r$-complete presentation $(\mathcal{S}, \mathcal{R})$ in the sense of [6]. Assume that for all $u \in \mathcal{S}$, there is $v \in \mathcal{S}$ such that $\mathcal{R}$ does not contain any relation of the form $u \cdots=v \cdots$. Also, suppose that $P$ embeds into a group $G$ such that $(G, P)$ is quasi-lattice ordered in the sense of [30]. Then $G_{0}=\{e\}$ and $\partial C_{\lambda}^{*}(P)$ is simple.

Proof. In view of Corollary 3.22, it suffices to prove $G_{0}=\{e\}$. Let $g \in G_{0}$. Assume that $g P \cap P \neq P$. Then $g \in G_{0}$ implies that this intersection is not empty. Hence, we must have $g P \cap P=p P$ for some $p \in P$ because $(G, P)$ is quasi-lattice ordered. As $p \neq e$, there exists $u \in \Sigma$ with $p P \subseteq u P$. By assumption, there exists $v \in \Sigma$ such that no relation in $R$ is of the form $u \cdots=v \cdots$. By r-completeness, $u P \cap v P=\emptyset$ (see [6, Proposition 3.3]), so that $g P \cap v P=\emptyset$. This contradicts $g \in G_{0}$. Hence, we must have $g P \cap P=P$, and similarly, $g^{-1} P \cap P=P$. These two equalities imply $g \in P^{*}$. But $P^{*}=\{e\}$ because $(G, P)$ is quasi-lattice ordered. Thus $g=e$. 
We would like to mention that results on simplicity of boundary quotients have been obtained in the special case of right LCM semigroups in [37, Theorem 4.12].

Remark 3.24. By going over to the opposite semigroup, we obtain analogous results for the right versions $C_{\rho}^{*}(P)$ and $\partial C_{\rho}^{*}(P)$.

Examples 3.25. Theorem 3.23 implies that for every graph-irreducible right-angled Artin monoid $A_{\Gamma}^{+}$in the sense of [4], $\partial C_{\lambda}^{*}\left(A_{\Gamma}^{+}\right)$is simple. Also, for the Thompson monoid $F^{+}=$ $\left\langle x_{0}, x_{1}, \ldots\right| x_{n} x_{k}=x_{k} x_{n+1}$ for $\left.k<n\right\rangle^{+}$, we get that $\partial C_{\rho}^{*}\left(F^{+}\right)$is simple.

Corollary 3.22 and [20, Corollary 5.10] imply that for every countable Krull ring $R$ with $\mathcal{P}(R)_{\text {inf }} \neq \emptyset$ or $\mathcal{P}(R)_{\text {fin }}$ infinite (see [20] for details), $\partial C_{\lambda}^{*}\left(R \rtimes R^{\times}\right)$is simple.

\section{Purely infinite Groupoids}

Our goal is to exhibit examples of purely infinite groupoids. More precisely, we study groupoids attached to graphs, groupoids corresponding to boundary quotients of semigroup $\mathrm{C}^{*}$-algebras, and groupoids underlying semigroup $\mathrm{C}^{*}$-algebras of $a x+b$-semigroups.

First of all, it is easy to see that for a partial dynamical system $G \curvearrowright X$, the transformation groupoid $G \ltimes X$ is purely infinite in the sense of [27] if and only if every compact open subset of $X$ is $(G, \mathcal{C O})$-paradoxical in the sense of [15, Definition 4.3], where $\mathcal{C O}$ is the set of compact open subsets of $X$.

The following lemma is easy to see, and will be used several times:

Lemma 4.1. Let $E$ be a semilattice. Every compact open subset $A \subseteq \widehat{E}$ can be written as a disjoint union $A=\bigsqcup_{i=1}^{m} U_{i}$ of basic open sets $U_{i}$ of the form

$$
U\left(e ; e_{1}, \ldots, e_{n}\right)=\left\{\chi \in \widehat{E}: \chi(e)=1, \chi\left(e_{1}\right)=\ldots=\chi\left(e_{n}\right)=0\right\}, e, e_{1}, \ldots, e_{n} \in E .
$$

This lemma will be helpful because given a partial system $G \curvearrowright \widehat{E}$ (or $G \curvearrowright X$ for any $G$ invariant subspace $X \subseteq \widehat{E}$ ), and we want to show that every compact open subset is $(G, \mathcal{C O}$ )paradoxical, then it suffices to show this for basic open sets of the form $U\left(e ; e_{1}, \ldots, e_{n}\right)$.

4.1. Graphs. Let us first recall a necessary and sufficient condition from [16], in terms of graphs, for pure infiniteness of graph $\mathrm{C}^{*}$-algebras. Let $\mathcal{E}=\left(\mathcal{E}^{0}, \mathcal{E}^{1}, r, s\right)$ be a graph, and we use the same notation as in $\S 3.2$. Note that since our notation differs from the one in [16] (see Remark 3.5), we have to reverse all the arrows in the condition for pure infiniteness.

For $v, w \in \mathcal{E}^{0}$, we write $w \leftarrow v$ if there exists a path $\mu \in \mathcal{E}^{*}$ from $v$ to $w$, i.e., with $r(\mu)=w$ and $s(\mu)=v$, and we write $w \& v$ if there is no such path. For $v \in \mathcal{E}^{0}$, let $\Omega(v)=\left\{w \in \mathcal{E}^{0}: w \neq v, w \nLeftarrow v\right\} . \quad v \in \mathcal{E}^{0}$ is called a breaking vertex if $\left|r^{-1}(v)\right|=\infty$ and $0<\left|r^{-1}(v) \backslash s^{-1}(\Omega(v))\right|<\infty$.

Moreover, we call $\mu \in \mathcal{E}^{*}$ a loop if $l(\mu) \geq 1$ and $r(\mu)=s(\mu)$. We say that $\mathcal{E}$ satisfies condition $(\mathrm{K})$ if for all $v \in \mathcal{E}^{0}$, whenever there exists a loop $\mu$ with $r(\mu)=v=s(\mu)$, there exists another loop $\mu^{\prime}$ with $r\left(\mu^{\prime}\right)=v=s\left(\mu^{\prime}\right)$ and $\mu \cdot \mu^{\prime}=0$ in $E_{\mathcal{E}}$.

Furthermore, a subset $M \subseteq \mathcal{E}^{0}$ is called a maximal tail if 
- for every $v \in \mathcal{E}^{0}$, whenever there is $w \in M$ with $v \leftarrow w$, then $v \in M$;

- for every $v \in M$ with $0<\left|r^{-1}(v)\right|<\infty$, there exists $\eta \in \mathcal{E}^{1}$ with $r(\eta)=v$ and $s(\eta) \in M$;

- for all $v, w \in M$, there exist $y \in M$ with $v \leftarrow y$ and $w \leftarrow y$.

We say that $v \in \mathcal{E}^{0}$ connects to a loop if there is a loop $\mu$ with $r(\mu)=w=s(\mu)$ with $v \leftarrow w$.

$\left[16\right.$, Theorem 2.3] says that $C^{*}(\mathcal{E})$ is purely infinite if and only if $\mathcal{E}$ satisfies the following condition:

(PI) There exist no breaking vertices in $\mathcal{E}, \mathcal{E}$ satisfies condition $(\mathrm{K})$, and every vertex in each maximal tail $M$ connects to a loop in $M$.

Lemma 4.2. Suppose that $\mathcal{E}$ satisfies condition $(P I)$. Let $v \in \mathcal{E}^{0}$ satisfy $\left|r^{-1}(v)\right|=\infty$. Then there exist infinitely many loops $\mu_{1}, \mu_{2}, \ldots \in \mathcal{E}^{*}$ with $r\left(\mu_{i}\right)=v=s\left(\mu_{i}\right)$ of the form $\mu_{i}=\zeta_{i} \eta_{i}$ for pairwise distinct $\zeta_{i} \in \mathcal{E}^{1}$.

Proof. Since $v$ is not a breaking vertex, we must have $\left|r^{-1}(v) \backslash s^{-1}(\Omega(v))\right| \in\{0, \infty\}$.

If $\left|r^{-1}(v) \backslash s^{-1}(\Omega(v))\right|=\infty$, then there exist infinitely many pairwise distinct $\zeta_{i} \in \mathcal{E}^{1}$ with $r\left(\zeta_{i}\right)=v$, and there is $\eta_{i} \in \mathcal{E}^{*}$ with $r\left(\eta_{i}\right)=s\left(\zeta_{i}\right), s\left(\eta_{i}\right)=v$. Then $\zeta_{i} \eta_{i}$ are the required loops.

If $\left|r^{-1}(v) \backslash s^{-1}(\Omega(v))\right|=0$, then consider the subset $M:=\left\{w \in \mathcal{E}^{0}: w \leftarrow v\right\}$. It is easy to see that $M$ is a maximal tail, with $v \in M$. By condition (PI), $v$ has to connect to a loop $\mu$ in $M$. This means that there is $w \in \mathcal{E}^{0}$ such that $w$ lies on $\mu$ and $v \leftarrow w$. But then, $w$ has to lie in $M$, so that $w \leftarrow v$ by definition of $M$; hence $v$ lies on a loop $v \leftarrow w \leftarrow v$. Let $\zeta_{1} \cdots \zeta_{n}$ be such a loop. Then $\zeta_{1} \in r^{-1}(v)$, but $\zeta_{1} \notin s^{-1}(\Omega(v))$ as $s\left(\zeta_{1}\right) \leftarrow v$, for instance via $\zeta_{2} \cdots \zeta_{n}$. Hence $0<\left|r^{-1}(v) \backslash s^{-1}(\Omega(v))\right|$, which is a contradiction.

Let $\mathbb{F} \ltimes \partial \mathcal{E}$ be the transformation groupoid attached to $\mathbb{F} \curvearrowright \partial \mathcal{E}$ as in $\S 3.2$.

Theorem 4.3. If $\mathcal{E}$ satisfies condition (PI), then $\mathbb{F} \ltimes \partial \mathcal{E}$ is purely infinite.

Proof. By Lemma 4.1, it suffices to show that basic open sets of the form $U\left(\mu ; \mu_{1}, \ldots, \mu_{n}\right)$ are $(\mathbb{F}, \mathcal{C O})$-paradoxical. By passing even further to finite unions if necessary, it suffices to treat the basic open sets $U\left(\mu ; \mu_{1}, \ldots, \mu_{n}\right)$ with $\left|r^{-1}(s(\mu))\right|=\infty$ (and $\left.\mu_{1}, \ldots, \mu_{n} \leq \mu\right)$, or basic open sets of the form $U(\mu)$.

Let us consider the first case. Set $U:=U\left(\mu ; \mu_{1}, \ldots, \mu_{n}\right)$. Let $v=s(\mu)$. Since $\left|r^{-1}(v)\right|=\infty$, Lemma 4.2 tells us that there are infinitely many loops $\mu_{1}, \mu_{2}, \ldots \in \mathcal{E}^{*}$ with $r\left(\mu_{i}\right)=v=s\left(\mu_{i}\right)$ of the form $\mu_{i}=\zeta_{i} \eta_{i}$ for pairwise distinct $\zeta_{i} \in \mathcal{E}^{1}$. Hence we can find, among the $\zeta_{i}$, edges $\zeta, \zeta^{\prime} \in \mathcal{E}^{1}$ with $r(\zeta)=r\left(\zeta^{\prime}\right)=v$ and $\mu \zeta \cdot \mu_{1}=\ldots=\mu \zeta \cdot \mu_{n}=0=\mu \zeta^{\prime} \cdot \mu_{1}=\ldots=\mu \zeta^{\prime} \cdot \mu_{n}=0$. Therefore, for $\chi \in \partial \mathcal{E}, \chi(\mu \zeta)=1$ implies $\chi\left(\mu_{i}\right)=0$ for all $1 \leq i \leq n$, so that $\chi \in U$, and similarly for $\zeta^{\prime}$. Moreover, $\mu \zeta \cdot \mu \zeta^{\prime}=0$ yields that $\chi(\mu \zeta)=1$ implies $\chi\left(\mu \zeta^{\prime}\right)=0$ and vice versa. Setting $g=\mu \zeta \mu^{-1} \in \mathbb{F}, h=\mu \zeta^{\prime} \mu^{-1} \in \mathbb{F}$, we obtain $g . U \subseteq U, h . U \subseteq U$ and $g . U \cap h . U=\emptyset$.

In the second case, let $v=s(\mu)$. By Lemma 4.2 and condition (PI), we can find $\alpha=$ $\alpha_{1} \cdots \alpha_{n} \in \mathcal{E}^{*}$ with $v=r(\alpha)$ and $\left|r^{-1}\left(\alpha_{i}\right)\right|<\infty(1 \leq i \leq n)$ such that there is a loop $\beta \in \mathcal{E}^{*}$ with $r(\beta)=s(\alpha)=s(\beta)$. Therefore, by passing to finite unions if necessary, we may assume by condition $(\mathrm{K})$ that there exist two loops $\zeta$ and $\zeta^{\prime}$ with $r(\zeta)=v=s(\zeta), r\left(\zeta^{\prime}\right)=v=s\left(\zeta^{\prime}\right)$ and $\zeta \cdot \zeta^{\prime}=0$. Again, setting $g=\mu \zeta \mu^{-1} \in \mathbb{F}, h=\mu \zeta^{\prime} \mu^{-1} \in \mathbb{F}$, we obtain $g . U \subseteq U, h . U \subseteq U$ and $g . U \cap h . U=\emptyset$.

It is clear that condition (PI) implies that $\mathbb{F} \curvearrowright \partial \mathcal{E}$ is residually topologically free, in the sense of [15, Definition 3.4 (ii)]. Moreover, [15, Theorem 4.4] tells us that if $\mathbb{F} \curvearrowright \partial \mathcal{E}$ is residually topologically free and if every compact open subset of $\partial \mathcal{E}$ is $(\mathbb{F}, \mathcal{C O})$-paradoxical, 
then $C^{*}(\mathcal{E}) \cong C(\partial \mathcal{E}) \rtimes_{r} \mathbb{F}$ must be purely infinite (using that $\mathbb{F}$ is free and [11, Theorem 22.9]). All in all, we obtain from Theorem 4.3:

Corollary 4.4. For a graph $\mathcal{E}, \mathbb{F} \curvearrowright \partial \mathcal{E}$ is residually topologically free and purely infinite if and only if $C^{*}(\mathcal{E})$ is purely infinite.

4.2. Boundary quotients of semigroup $\mathrm{C}^{*}$-algebras. Let $P$ be a subsemigroup of a group $G$. Let $E$ be the semilattice of idempotents of $S=I_{l}(P)$. We write $\Omega$ for $\Omega_{P}, \Omega_{\max }$ for $\widehat{E}_{\max }$ and $\partial \Omega$ for $\partial \Omega_{P}=\partial \widehat{E}$.

Theorem 4.5. $G \ltimes \partial \Omega$ is purely infinite if and only if there exist $p, q \in P$ with $p P \cap q P=\emptyset$.

Proof. Obviously, if $p P \cap q P \neq \emptyset$ for all $p, q \in P$, then $\partial \Omega$ degenerates to a point. For the converse, let $U=\left\{\psi \in \partial \Omega: \psi(X)=1, \psi\left(X_{1}\right)=\ldots=\psi\left(X_{n}\right)=0\right\}$ be a basic open subset for some $X, X_{1}, \ldots, X_{n} \in \mathcal{J}$. By Lemma 4.1, it suffices to show that $U$ is $(G, \mathcal{C O})$-paradoxical. Since $\Omega_{\max }$ is dense in $\partial \Omega$, there exists $\chi \in \Omega_{\max }$ with $\chi \in U$. As $\chi$ lies in $\Omega_{\max }, \chi\left(X_{i}\right)=0$ implies that there exists $Y_{i} \in \mathcal{J}$ with $X_{i} \cap Y_{i}=\emptyset$ and $\chi\left(Y_{i}\right)=1$. Let $Y:=X \cap \bigcap_{i=1}^{n} Y_{i}$. Certainly, $Y \neq \emptyset$ as $\chi(Y)=1$. Moreover, for every $\psi \in \partial \Omega, \psi(Y)=1$ implies $\psi \in U$. Now choose $x \in Y$. By assumption, we can find $p, q \in P$ with $p P \cap q P=\emptyset$. For $\psi \in \partial \Omega$, $x p . \psi(x p P)=\psi(P)=1$. Similarly, for all $\psi \in \partial \Omega, x q . \psi(x q P)=1$. Thus $x p . U \subseteq x p . \partial \Omega \subseteq U$, $x q . U \subseteq x q . \partial \Omega \subseteq U$ and $(x p . U) \cap(x q . U) \subseteq(x p . \partial \Omega) \cap(x q . \partial \Omega)=\emptyset$ since $x p P \cap x q P=\emptyset$.

This strengthens and explains [19, Corollary 7.23]. It also generalizes [37, Theorem 4.15].

4.3. Groupoids from $a x+b$-semigroups. Let $R$ be an integral domain with quotient field $K$. Consider the $a x+b$-semigroup $R \rtimes R^{\times}$, viewed as a subsemigroup of $K \rtimes K^{\times}$. Let $E$ be the semilattice of idempotents in $S=I_{l}\left(R \rtimes R^{\times}\right)$.

Theorem 4.6. Let $R$ be an integral domain with $\operatorname{Jac}(R)=(0)$ and $R^{\times} \neq R^{*}$. Then $(K \rtimes$ $\left.K^{\times}\right) \ltimes \widehat{E}$ is purely infinite.

Proof. Let $U$ be the basic open subset

$$
\begin{aligned}
& U\left(x+I ; x_{1}+I_{1}, \ldots, x_{n}+I_{n}\right) \\
= & \left\{\chi \in \widehat{E}: \chi\left((x+I) \times I^{\times}\right)=1 ; \chi\left(\left(x_{1}+I_{1}\right) \times I_{1}^{\times}\right)=\ldots=\chi\left((x+I) \times I^{\times}\right)=0\right\},
\end{aligned}
$$

where $I_{i} \subseteq I \subsetneq R$. By Lemma 4.1, it suffices to show that $U$ is $\left(K \rtimes K^{\times}, \mathcal{C O}\right)$-paradoxical.

Set $J:=\bigcap_{i=1}^{n} I_{i}$. Obviously, $J \neq(0)$. Therefore $1+J \nsubseteq R^{*}$. Otherwise, by [2, Proposition 1.9], $J \subseteq \operatorname{Jac}(R)=(0)$, which is a contradiction. So we can choose $a \in(1+J) \backslash R^{*}, a \neq 0$, as $R^{\times} \neq R^{*}$. Then $J \nsubseteq a R$. Otherwise, there would exist $r \in R$ with $a-1=a r$, so that $a(1-r)=1$ contradicting $a \notin R^{*}$. Choose $b_{1} \in J, \delta \in J \backslash a R$ and set $b_{2}:=b_{1}+\delta$.

For $k=1,2,\left(b_{k}, a\right) \cdot U=U\left(b_{k}+a x+a I ; b_{k}+a x_{1}+a I_{1}, \ldots, b_{k}+a x_{n}+a I_{n}\right)$. Since $b_{k}+a x+a I \subseteq$ $a x+a I+J \subseteq a(x+I)+J \subseteq(1+J)(x+I)+J \subseteq x+I$, every $\chi \in\left(b_{k}, a\right) \cdot U$ satisfies $\chi\left((x+I) \times I^{\times}\right)=1$. Moreover, we have $\left(x_{i}+I_{i}\right) \cap\left(b_{k}+a x+a I\right)=b_{k}+\left(x_{i}+I_{i}\right) \cap(a x+a I)=$ $b_{k}+\left(a x_{i}+I_{i}\right) \cap\left(a x_{i}+a I\right)$ since $x_{i}-a x_{i}=(1-a) x_{i} \in J$.

We claim that $I_{i} \cap a I=a I_{i}$. "" is clear. If $r \in I_{i} \cap a I$, then $r=a s$ for some $s \in I$, so that $s=(1-a+a) s=(1-a) s+a s \in J+I_{i} \subseteq I_{i}$. This proves " $\subseteq$ ".

Hence $\left(x_{i}+I_{i}\right) \cap\left(b_{k}+a x+a I\right)=b_{k}+a x_{i}+a I_{i}$. Therefore, every $\chi \in\left(b_{k}, a\right) . U$ satisfies $\chi\left(\left(x_{i}+I_{i}\right) \times I_{i}^{\times}\right)=0$. This shows that for $k=1,2,\left(b_{k}, a\right) . U \subseteq U$. 
Also, $\left(b_{1}+a x+a I\right) \cap\left(b_{2}+a x+a I\right)=\emptyset$ as $b_{2}-b_{1}=\delta \notin a R$. Hence $\left(b_{1}, a\right) \cdot U \cap\left(b_{2}, a\right) \cdot U=\emptyset$.

This strengthens and explains [20, Theorem 1.3].

4.4. Almost finite groupoids. Apart from purely infinite groupoids, Matui also introduced almost finite ones in [26]. We would like to end with the following obervation concerning the relation between almost finiteness and amenability:

Proposition 4.7. Let $G$ be a group acting on a compact space $X$. Assume that there exists $x \in X$ with trivial stabilizer group, i.e., $G_{x}=\{g \in G: g \cdot x=x\}=\{e\}$. If the transformation groupoid $G \ltimes X$ is almost finite, then $G$ is amenable.

Proof. Let $E \subseteq G$ be a finite subset with $E=E^{-1}$. For every $\varepsilon>0$, we have to find a finite subset $F \subseteq G$ such that for every $s \in E,|s F \triangle F| /|F|<\varepsilon$.

Set $C:=E \times X \subseteq G \ltimes X$. Let $x \in X$ be as above, i.e., $\{g \in G: g \cdot x=x\}=\{e\}$. Since $G \ltimes X$ is almost finite, we can find an elementary subgroupoid $K$ of $G \ltimes X$ such that $|C K x \backslash K x| /|K x|<\frac{\varepsilon}{2}$. Let $F=\{g \in G:(g, x) \in K\}$. F is contained in the image of $K$ under the canonical projection $G \ltimes X \rightarrow G,(g, x) \mapsto g$. Therefore, $F$ is a finite subset of $G$. We have $K x=\{(g, x): g \in F\}$ and $C K x \backslash K x=\{(g, x): g \in E F \backslash F\}=\bigcup_{s \in E}\{(g, x): g \in s F \backslash F\}$. Therefore, $\frac{\varepsilon}{2}>|C K x \backslash K x| /|K x| \geq|s F \backslash F| /|F|$ for all $s \in E$. For $s \in E, F \backslash s F=$ $F \backslash(F \cap s F)=s\left(s^{-1} F \backslash\left(s^{-1} F \cap F\right)=s\left(s^{-1} F \backslash F\right)\right.$. Hence $|F \backslash s F|=\left|s^{-1} F \backslash F\right|<\frac{\varepsilon}{2}|F|$ by our computation above. (Note that by assumption, $E=E^{-1}$.)

Therefore, for every $s \in E,|s F \triangle F| /|F|=|s F \backslash F| /|F|+|F \backslash s F| /|F|<\varepsilon$.

\section{REFERENCES}

[1] F. ABADIE, On partial actions and groupoids, Proc. Am. Math. Soc. 132 (2003), 1037-1047.

[2] M.F. Atiyah and I.G. MacDonald, Introduction to Commutative Algebra, Addison-Wesley Publishing Company, Reading, Massachusetts, 1969.

[3] N. Brownlowe, T. Carlsen and M. Whittaker, Graph algebras and orbit equivalence, to appear in Ergod. Th. Dyn. Syst.

[4] J. CRISP and M. LACA, Boundary quotients and ideals of Toeplitz algebras of Artin groups, J. Funct. Anal. 242 (2007), 127-156.

[5] J. Cuntz, S. Echterhoff and X. LI, On the K-theory of crossed products by automorphic semigroup actions, Quart. J. Math. 64 (2013), 747-784.

[6] P. Dehornoy, Complete positive group presentations, Journal of Algebra 268 (2003), $156-197$.

[7] R. Exel, Amenability for Fell bundles, J. Reine Angew. Math. 492 (1997), 41-73.

[8] R. ExeL, Inverse semigroups and combinatorial $C^{*}$-algebras, Bull. Braz. Math. Soc. (N.S.) 39 (2008), 191-313.

[9] R. ExEL, Tight representations of semilattices and inverse semigroups, Semigroup Forum 79 (2009), 159182.

[10] R. ExEL, Reconstructing a totally disconnected groupoid from its ample semigroup, Proc. Am. Math. Soc. 138 (2010), 2991-3001.

[11] R. Exel, Partial Dynamical Systems, Fell Bundles and Applications, available on R. Exel's homepage.

[12] R. Exel, D. Goncalves and C. Starling, The tiling $C^{*}$-algebra viewed as a tight inverse semigroup algebra, Semigroup Forum 84 (2012), 229-240.

[13] R. ExEL and M. LACA, Cuntz-Krieger algebras for infinite matrices, J. reine angew. Math. 512 (1999), 119-172. 
[14] R. Exel, M. LACA and J. Quigg, Partial dynamical systems and $C^{*}$-algebras generated by partial isometries, J. Op. Th. 47 (2002), 169-186.

[15] T. Giordano and A. Sierakowski, Purely infinite partial crossed products, J. Funct. Anal. 266 (2014), $5733-5764$.

[16] J.H. Hoang and W. Syzmanski, Purely infinite Cuntz-Krieger algebras of directed graphs, Bull. London Math. Soc. 35 (2003), 689-696.

[17] M. Hochster, Subsemigroups of amenable groups, Proc. Amer. Math. Soc. 21 (1969), 363-364.

[18] X. LI, Semigroup C*-algebras and amenability of semigroups, J. Funct. Anal. 262 (2012), 4302-4340.

[19] X. LI, Nuclearity of semigroup $C^{*}$-algebras and the connection to amenability, Adv. in Math. 244 (2013), 626-662.

[20] X. LI, Semigroup C*-algebras of ax + b-semigroups, Trans. Amer. Math. Soc. 368 (2016), $4417-4437$.

[21] X. LI, Continuous orbit equivalence rigidity, preprint, arXiv:1503.01704.

[22] X. Li and M. D. NorLing, Independent resolutions for totally disconnected dynamical systems I: Algebraic case, J. Alg. 424 (2015), 98-125.

[23] X. Li and M.D. Norling, Independent resolutions for totally disconnected dynamical systems II: $C^{*}$ algebraic case, J. Op. Th. 75 (2016), 163-193.

[24] K. Matsumoto, Orbit equivalence of topological Markov shifts and Cuntz-Krieger algebras, Pacific J. Math. $246(2010), 199-225$.

[25] K. Matsumoto and H. Matui, Continuous orbit equivalence of topological Markov shifts and CuntzKrieger algebras, Kyoto J. Math. 54 (2014), 863-877.

[26] H. MAtui, Homology and topological full groups of étale groupoids on totally disconnected spaces, Proc. Lond. Math. Soc. 104 (2012), 27-56.

[27] H. Matui, Topological full groups of one-sided shifts of finite type, J. Reine Angew. Math. 705 (2015), $35-84$.

[28] K. McClanahan, K-theory for partial crossed products by discrete groups, J. Funct. Anal. 130 (1995), $77-117$.

[29] D. Milan and B. Steinberg, On inverse semigroup $C^{*}$-algebras and crossed products, Groups Geom. Dyn. 8 (2014), 485-512.

[30] A. NiCA, $C^{*}$-algebras generated by isometries and Wiener-Hopf operators, J. Operator Theory 27 (1992), $17-52$.

[31] M.D. Norling, Inverse semigroup $C^{*}$-algebras associated with left cancellative semigroups, Proc. Edinb. Math. Soc. (Series 2) 57 (2014), 533-564.

[32] A.L.T. Paterson, Groupoids, Inverse Semigroups, and their Operator Algebras, Progress in mathematics 170, Birkhäuser, Boston, 1999.

[33] J. Renault, A groupoid approach to $C^{*}$-algebras, LNM 793, Springer, Berlin Heidelberg New York, 1980.

[34] J. Renault, Cartan subalgebras in $C^{*}$-algebras, Irish Math. Soc. Bull. 61 (2008), $29-63$.

[35] J. Renault, $C^{*}$-algebras and Dynamical Systems, Publicacoes matematicas, IMPA, 2009.

[36] M. RøRdam and A. Sierakowski, Purely infinite $C^{*}$-algebras arising from crossed products, Ergod. Th. Dyn. Syst. 32 (2012), 273-293.

[37] C. Starling, Boundary quotients of $C^{*}$-algebras of right LCM semigroups, J. Funct. Anal. 268 (2015), $3326-3356$

[38] S.B.G. Webster, The path space of a directed graph, Proc. Amer. Math. Soc. 142 (2014), 213-225.

Xin Li, School of Mathematical Sciences, Queen Mary University of London, Mile End Road, LONDON E1 4NS, UNITED KINGDOM

E-mail address: xin.li@qmul.ac.uk 